\title{
A retrial queueing-inventory system with $J$-additional options for service and finite source
}

\author{
VSS Yadavalli* \\ K Jeganathan ${ }^{\dagger}$ \\ T Venkatesan $\ddagger$ \\ S Padmasekaran ${ }^{\S}$ \\ S Jehoashan Kingsly
}

Received: 25 August 2016; Revised: 17 May 2017; Accepted: 9 November 2017

\begin{abstract}
A continuous review $(s, S)$ inventory system at a service facility with finite homogeneous sources of demands and retrial is analysed. The lifetime of each item is assumed to be exponential. Before items are delivered to the customers, some basic service on the item must be performed. It is known as a regular or main service. The service may get interrupted according to a Poisson process and it restarts after an exponentially distributed time. If the server is idle at the time of arrival of a customer and the inventory level is positive, then the service begins immediately. After the completion of regular service, a customer may either abandon the system forever or demand for a second service from the same server, which is multi-optional. If any arriving customer finds that the server is busy or inventory level is zero, he/she either enters into the orbit with probability $p$ or balks (does not enter) with probability $1-p$. The stationary distribution of the number of customers in the system, server status and the inventory level is obtained by the matrix method. The Laplace-Stieltjes transform of the waiting time of the tagged customer is derived. Various system performance measures are derived and the total expected cost rate is computed under a suitable cost structure. A numerical illustration is given.
\end{abstract}

Key words: $\quad(s, S)$ policy, service interruption, finite source, retrial, repair, essential and optional service.

\section{Introduction}

In recent years, there has been a considerable interest in the stochastic inventory system in which an item demanded by the customer is not immediately delivered. This situation

*Department of Industrial and Systems Engineering, University of Pretoria, Pretoria, South Africa

${ }^{\dagger}$ Corresponding author: Ramanujan Institute for Advanced Study in Mathematics, University of Madras, Chennai, India, email: jegan.nathan85@yahoo.com

${ }^{\ddagger}$ Department of Mathematics, RMD Engineering College, Chennai, India.

${ }^{\S}$ Department of Mathematics, Periyar University, Salem, India.

ॠDepartment of Mathematics, Adhiyamaan College of Engineering, Hosur, India. 
arises when the items in the inventory needs some time for preparation and hence it is considered as having positive service time. Sigman and Simchi-Levi [17] introduced the notion of inventory with positive service time. They assumed that the service time follows an arbitrary distribution and the customers arrive according to a Poisson process wherein the demand is for a single item per customer. Berman et al. [3] formulated an inventory model where both demand and service rates are assumed to be deterministic and constant. Later Berman and Kim [4] considered a service facility that provides service to customers using items of inventory with the assumption of Poisson arrival, exponential service times and zero lead times. Berman and Sapna [6] studied a finite capacity inventory model with Poisson arrival, arbitrarily distributed service times and zero lead times. Berman and Kim [5] analysed a queueing-inventory system for service facilities with unlimited waiting space for customers. Maike Schwarz et al. [16] have considered a M/M/1 queueing system with attached inventory. They assumed a Poisson demand, exponentially distributed lead times and lost sales for infinite and finite waiting rooms.

In all previously mentioned papers, the researchers analysed a queueing inventory model with a service facility and infinite number of sources. Nevertheless, in many real life situations, it is important to take into account the fact that the number of customers in the source decreases as the number of customers in the system increases. This leads to the study of the inventory model with a finite number of sources. In this paper, a single server queueing-inventory system with a finite number of sources is discussed. A continuous review perishable inventory model with finite population was first initiated by Sivakumar [18]. He assumed that the customers arriving at a stock-out period enter into the orbit and they retry after some random time. The lifetime of each item follows an exponential distribution. Following this, a number of papers on inventory models with a finite population have emerged. Multi-server retrial inventory system with a finite number of sources was studied by Yadavalli et al. [24] in which the customers arrive according to a quasi random process. The service times and the lead times were assumed to be exponential. Shophia Lawrence et al. [15] analysed a service facility with a finite population in which items in inventory are perishable and customer demand is satisfied only after performing some service on it. The service time and the lead time have a Phase type distribution. The lifetime of each item follows a negative exponential distribution.

Many researchers have studied retrial queueing systems with a finite and infinite number of sources extensively. Artalejo [1], Falin \& Artalejo [7] and Falin \& Templeton [8] provide reviews on this queueing system. A numerical illustration of inventory systems with retrial was studied by Artalejo et al. [2]. The study of inventory models with server interruptions is a topic that has received considerable attention in the last decade. The inventory model with instantaneous replenishment is discussed by Krishnamoorthy et al. [12] wherein the service is subject to interruptions. Krishnamoorthy et al. [13] made an extensive study of an $(s, S)$ inventory model with the assumption of Poisson arrival and exponential service time. According to a Poisson process the service may get interrupted and it restarts after an exponentially distributed time. Yadavalli et al. [22] analysed a finite source perishable inventory system with a service facility having two heterogeneous servers and repeated attempts. They assumed that the first server is perfectly reliable and the second server is subject to interruptions. 
From the above papers, the customer will move away from the system after completion of regular or essential service. However, in day-to-day life, it does not always happen. In some queueing situations all arriving customers require the essential service, whereas a few of them may further demand the subsidiary service provided by the same server immediately after completion of the regular service. Several researchers have studied the concept of additional optional service with queues [11, 19, 20]. Jeganathan [9] investigated a continuous review perishable $(s, S)$ inventory system with $N$ optional services, in which some of the arriving customers asked for second optional service as soon as the completion of first essential service and the second service is multi-optional. He assumed that the customer arrivals follow a Poisson process. Yadavalli \& Jeganathan [23] analysed a finite source perishable inventory system with second optional service and server interruptions. Recently, Jeganathan et al. [10] analysed a retrial queueing-inventory system with priority customers and second optional service.

A finite population Markovian inventory system with server interruptions, multi-optional service and repeated attempts is considered in this paper. The rest of the paper is organized as follows. A detailed description of the model is explained in $\S 2$. In $\S 3$, the mathematical solution of the model is carried out. The Laplace-Stieltjes transform of waiting time distribution for customers in the orbit is derived in $\S 4$. Some key system performance measures are obtained in $\S 5$, while $\S 6$ is dedicated to cost analysis and sensitivity investigation. Conclusions are given in the final section.

\section{Model description}

In this investigation, a finite source Markovian inventory system is considered with the following assumptions. Consider a single server perishable inventory system, where the primary arrivals are generated from $N, 1<N<\infty$ homogeneous sources. The inventory is replenished according to $(s, S)$ ordering policy. According to this, an order for $Q(=$ $S-s>s+1)$ items are placed when the on-hand inventory level falls below $s$. The requirement $S-s>s+1$ assures that after a replenishment, the inventory level will be greater than the reorder level. Otherwise it is impossible to place a reorder which leads to perpetual shortage. The positive lead-time of the replenishment is assumed to be negative exponential with the rate $\beta(>0)$. The lifetime of each commodity has a negative exponential distribution with the parameter $\gamma(>0)$. It is assumed that during service, the items in inventory cannot be perished.

The server can retain in three states, namely idle, busy and interruption. Likewise, each source can also be in three states, namely free, retrial and under service.

1. If a source is in free state at time $t$ a primary customer can arrive during the interval $(t, t+d t)$ with probability $\lambda d t+o(t)$. It is assumed that the waiting space is not available in front of the server. When the server is in idle state and the inventory level is positive then the demand is served immediately. Hence, the source moves into "under service" state and the server moves into "busy" state.

2. If the demand finds the inventory level zero or server busy or server is on interruption at the moment of their arrival, then they either abandon (with non-zero probability 
p) the area of service and join a pool of blocked customers called orbit or balk the system (with probability $1-p$ ).

In this paper, the classical retrial method is followed. More explicitly, the probability of a repeated attempt in an interval $(t, t+d t)$ given that $i$ customers are in the orbit attempt is $i \theta+o(d t)$. If the server is idle with positive inventory then it provides the first essential service (FES) to all arriving customers. The first essential service is also referred to as regular service or main service. It is assumed to be exponentially distributed with parameter $\mu_{0}$. As soon as the first essential service of each demand is completed, then with probability $r_{k}, 1 \leq k \leq J$, a customer may opt to receive a second optional service (SOS) from $J(J \geq 1)$ kinds of different services (i.e., Type1, Type2,..., Type $J$ ), or else, with probability $r_{0}=1-\sum_{k=1}^{J} r_{k}$ the customer may opt to abandon the system and then the server becomes idle. The service time of the second optional service is assumed to be distributed exponentially with parameter $\mu_{k}, k=1,2, \ldots, J$.

When the server provides the first essential service to a customer, the service may get interrupted according to a Poisson process of rate $\alpha_{0}$. In the second multi-optional service, the service may get interrupted with an exponential rate $\alpha_{k}, 1 \leq k \leq J$. It is assumed that while the server is under interruption, no further interruption can befall the server. The repair time of both service phases (first essential service and second multi- optional service) are assumed to be exponentially distributed with parameters $\eta_{0}$ and $\eta_{k}, 1 \leq k \leq J$, respectively. Figure 1 shows a typical picture of the model. All random variables are independent of each other.

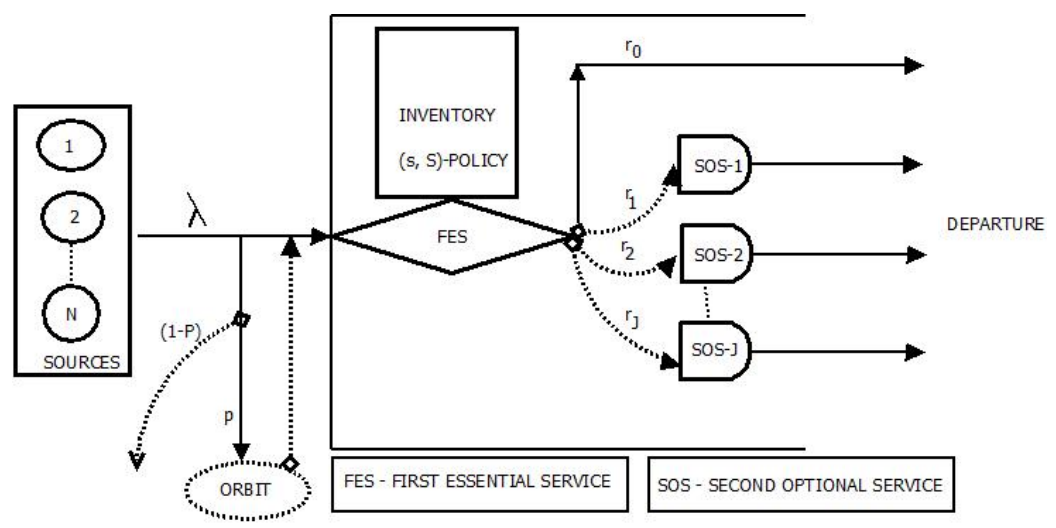

Figure 1: Dynamics of the queueing inventory system with finite source.

\section{Mathematical formulation of the model}

Let $l(t)$ and $x(t)$ indicate the inventory level and the number of customers in the orbit at time $t, l(t) \in\{0,1,2, \ldots, S\}$ and $x(t) \in\{0,1,2, \ldots, N\}$, and let $y(t)$ denote the status of the server by 
$y(t)= \begin{cases}S_{0}, & \text { if the server is idle at time } t, \\ S_{1}, & \text { if the server is busy with FES at time } t, \\ S_{2}, & \text { if the server is on interruption during FES at time } t, \\ 1, & \text { if the server is busy with Type } 1 \text { service at time } t, \\ 2, & \text { if the server is busy with Type } 2 \text { service at time } t, \\ \vdots & \vdots \\ J-1, & \text { if the server is busy with Type } J-1 \text { service at time } t, \\ J, & \text { if the server is busy with Type } J \text { service at time } t, \\ J+1, & \text { if the server is on interruption during Type } 1 \text { service at time } t, \\ J+2, & \text { if the server is on interruption during Type } 2 \text { service at time } t, \\ \vdots & \vdots \\ 2 J-1, & \text { if the server is on interruption during Type } J-1 \text { service at time } t, \\ 2 J, & \text { if the server is on interruption during Type } J \text { service at time } t .\end{cases}$

The activities of the system can be expressed by a three-dimensional stochastic process $\{a(t)=(l(t), y(t), x(t)), t \geq 0\}$, with the finite discrete state space $E$, where

$$
\begin{aligned}
E= & \left\{0 \leq i_{1} \leq S, i_{2}=S_{0}, 0 \leq i_{3} \leq N\right\} \cup \\
& \left\{1 \leq i_{1} \leq S, i_{2}=S_{1}, S_{2}, 0 \leq i_{3} \leq N-1\right\} \cup \\
& \left\{0 \leq i_{1} \leq S, 1 \leq i_{2} \leq 2 J, 0 \leq i_{3} \leq N-1\right\}
\end{aligned}
$$

Due to the assumptions of a Poisson arrival process, exponentially distributed service times, the replenishment process, the repair process and the interruption process $\{a(t), t \geq$ $0\}$ is a Homogeneous Continuous Time Markov Chain (HCTMC). Its limiting distribution is indicated by $\pi\left(i_{1}, i_{2}, i_{3}\right)$ :

$$
\pi^{\left(i_{1}, i_{2}, i_{3}\right)}=\lim _{t \rightarrow \infty} \operatorname{Pr}\left[l(t)=i_{1}, y(t)=i_{2}, x(t)=i_{3} \mid l(0), y(0), x(0)\right] .
$$

In the sequel, $I_{k}$ refers to an identity matrix of order $k$, e refers to a column vector of appropriate dimension containing all ones, $[A]_{i j}$ denotes the entry at $(i, j)^{\text {th }}$ position of a matrix $A, \delta$ is the delta function defined by $\delta_{i j}=1$ if $i=j$, otherwise $\delta_{i j}=0, H(x)$ is the Heaviside function i.e. $H(x)=1$ if $x \geq 0$, otherwise $H(x)=0$ and $k \in V_{i}^{j}$ denotes $k=i, i+1, \ldots j$.

The steady-state equation of $\{a(t)=(l(t), y(t), x(t)), t \geq 0\}$ satisfies the following balance equations.

For $i_{1} \in V_{0}^{S}, \quad i_{2}=S_{0}$, and $i_{3} \in V_{0}^{N}$,

$$
\begin{array}{r}
\left(\left(N-i_{3}\right) \lambda+H\left(s-i_{1}\right) \beta+i_{1} \gamma+\bar{\delta}_{i_{1} 0} i_{3} \theta\right) \pi^{\left(i_{1}, i_{2}, i_{3}\right)}=\bar{\delta}_{i_{1} S}\left(i_{1}+1\right) \gamma \pi^{\left(i_{1}+1, i_{2}, i_{3}\right)}+ \\
\bar{\delta}_{i_{3} 0} \delta_{i_{1} 1} p\left(N-i_{3}\right) \lambda \pi^{\left(i_{1}-1, i_{2}, i_{3}-1\right)}+H\left(i_{1}-Q\right) \beta \pi^{\left(i_{1}-S+s, i_{2}, i_{3}\right)}+ \\
\bar{\delta}_{i_{3} N} \bar{\delta}_{i_{1} S} r_{0} \mu_{0} \pi^{\left(i_{1}+1, S_{1}, i_{3}\right)}+\bar{\delta}_{i_{3} N} \sum_{k=1}^{J} \mu_{k} \pi^{\left(i_{1}, k, i_{3}\right)}
\end{array}
$$


For $i_{1} \in V_{1}^{S}, \quad i_{2}=S_{1}$, and $i_{3} \in V_{0}^{N-1}$,

$$
\begin{array}{r}
\left(p\left(N-\left(i_{3}+1\right)\right) \lambda+H\left(s-i_{1}\right) \beta+\bar{\delta}_{i_{1} 1}\left(i_{1}-1\right) \gamma+\sum_{k=0}^{J} r_{k} \mu_{0}+\alpha_{0}\right) \pi^{\left(i_{1}, i_{2}, i_{3}\right)}= \\
\bar{\delta}_{i_{3} 0} p\left(N-i_{3}\right) \lambda \pi^{\left(i_{1}, i_{2}, i_{3}-1\right)}+H\left(i_{1}-(Q+1)\right) \beta \pi^{\left(i_{1}-S+s, i_{2}, i_{3}\right)}+ \\
\bar{\delta}_{i_{1} S} i_{1} \gamma \pi^{\left(i_{1}+1, i_{2}, i_{3}\right)}+\left(i_{3}+1\right) \theta \pi^{\left(i_{1}+1, S_{0}, i_{3}+1\right)}+\left(N-i_{3}\right) \lambda \pi^{\left(i_{1}, S_{0}, i_{3}\right)}+\eta_{0} \pi^{\left(i_{1}, S_{2}, i_{3}\right)} .
\end{array}
$$

For $i_{1} \in V_{1}^{S}, \quad i_{2}=S_{2}$, and $i_{3} \in V_{0}^{N-1}$,

$$
\begin{array}{r}
\left(p\left(N-\left(i_{3}+1\right)\right) \lambda+H\left(s-i_{1}\right) \beta+\bar{\delta}_{i_{1} 1}\left(i_{1}-1\right) \gamma+\eta_{0}\right) \pi^{\left(i_{1}, i_{2}, i_{3}\right)}=\bar{\delta}_{i_{3} 0} p\left(N-i_{3}\right) \lambda \pi^{\left(i_{1}, i_{2}, i_{3}-1\right)} \\
+H\left(i_{1}-(Q+1)\right) \beta \pi^{\left(i_{1}-S+s, i_{2}, i_{3}\right)}+\bar{\delta}_{i_{1} S} i_{1} \gamma \pi^{\left(i_{1}+1, i_{2}, i_{3}\right)}+\alpha_{0} \pi^{\left(i_{1}, S_{1}, i_{3}\right)}
\end{array}
$$

For $i_{1} \in V_{0}^{S}, \quad i_{2} \in V_{1}^{J}$, and $i_{3} \in V_{0}^{N-1}$,

$$
\begin{array}{r}
\left(p\left(N-\left(i_{3}+1\right)\right) \lambda+H\left(s-i_{1}\right) \beta+\bar{\delta}_{i_{1} 0} i_{1} \gamma+\sum_{k=1}^{J}\left(\delta_{i_{2} k} \mu_{k}\right)+\sum_{k=1}^{J}\left(\delta_{i_{2} k} \alpha_{k}\right)\right) \pi^{\left(i_{1}, i_{2}, i_{3}\right)} \\
\bar{\delta}_{i_{3} 0} p\left(N-i_{3}\right) \lambda \pi^{\left(i_{1}, i_{2}, i_{3}-1\right)}+ \\
+H\left(i_{1}-Q\right) \beta \pi^{\left(i_{1}-S+s, i_{2}, i_{3}\right)}+\bar{\delta}_{i_{1} S}\left(i_{1}+1\right) \gamma \pi^{\left(i_{1}+1, i_{2}, i_{3}\right)} \\
+\bar{\delta}_{i_{1} 0}\left\{\sum_{k=1}^{J} r_{k} \mu_{0}\right\} \pi^{\left(i_{1}, S_{1}, i_{3}\right)}+\left\{\sum_{k=1}^{J} \delta_{i_{2} J+k} \eta_{k}\right\} \pi^{\left(i_{1}, J+k, i_{3}\right)}
\end{array}
$$

For $i_{1} \in V_{0}^{S}, \quad i_{2} \in V_{J+1}^{2 J}$, and $i_{3} \in V_{0}^{N-1}$,

$$
\begin{aligned}
& \left(p\left(N-\left(i_{3}+1\right)\right) \lambda+H\left(s-i_{1}\right) \beta+\bar{\delta}_{i_{1} 0} i_{1} \gamma+\sum_{k=1}^{J}\left(\delta_{i_{2} k} \eta_{k}\right)\right) \pi^{\left(i_{1}, i_{2}, i_{3}\right)}= \\
& \bar{\delta}_{i_{3} 0} p\left(N-i_{3}\right) \lambda \pi^{\left(i_{1}, i_{2}, i_{3}-1\right)}+H\left(i_{1}-Q\right) \beta \pi^{\left(i_{1}-S+s, i_{2}, i_{3}\right)}+\bar{\delta}_{i_{1} S}\left(i_{1}+1\right) \gamma \pi^{\left(i_{1}+1, i_{2}, i_{3}\right)} \\
& +\left\{\sum_{k=J+1}^{2 J} \delta_{i_{2} k} \alpha_{\left(i_{2}-J\right)}\right\} \pi^{\left(i_{1}, i_{2}-J, i_{3}\right)} .
\end{aligned}
$$

To mark the steady-state equations in expressions of matrix form, states in $E$ are rescheduled in the following lexicographical ordering:

$$
\begin{aligned}
& \lll i_{1} \ggg= \begin{cases}\ll i_{1}, i_{2}, \gg, & i_{1}=1,2, \ldots S, i_{2}=S_{0}, S_{1}, S_{2}, 1,2, \ldots, 2 J ; \\
\ll i_{1}, i_{2}, \gg, & i_{1}=0, i_{2}=S_{0}, 1,2, \ldots, 2 J ;\end{cases} \\
& \ll i_{1}, i_{2} \gg= \begin{cases}<i_{1}, S_{0}, 0>,<i_{1}, S_{0}, 1>, \ldots,<i_{1}, S_{0}, N>, & i_{1}=0,1,2, \ldots S ; \\
<i_{1}, i_{2}, 0>,<i_{1}, i_{2}, 1>, \ldots,<i_{1}, i_{2}, N-1>, & i_{1}=1,2, \ldots S, \\
& i_{2}=S_{1}, S_{2} ; \\
<i_{1}, i_{2}, 0>,<i_{1}, i_{2}, 1>, \ldots,<i_{1}, i_{2}, N-1>, & i_{1}=0,1,2, \ldots S, \\
& i_{2}=1,2, \ldots, 2 J ;\end{cases}
\end{aligned}
$$


and define the corresponding vectors

$$
\begin{aligned}
\boldsymbol{\Pi}^{(\mathbf{0})} & =\left(\boldsymbol{\Pi}^{\left(\mathbf{0}, \mathbf{S}_{\mathbf{0}}\right)}, \boldsymbol{\Pi}^{(\mathbf{0}, \mathbf{1})}, \boldsymbol{\Pi}^{(\mathbf{0}, \mathbf{2})}, \ldots, \boldsymbol{\Pi}^{(\mathbf{0}, \mathbf{2} \mathbf{J})}\right), \\
\boldsymbol{\Pi}^{\left(\mathbf{i}_{\mathbf{1}}\right)} & =\left(\boldsymbol{\Pi}^{\left(\mathbf{i}_{\mathbf{1}}, \mathbf{S}_{\mathbf{0}}\right)}, \boldsymbol{\Pi}^{\left(\mathbf{i}_{\mathbf{1}}, \mathbf{S}_{\mathbf{1}}\right)}, \boldsymbol{\Pi}^{\left(\mathbf{i}_{\mathbf{1}}, \mathbf{S}_{\mathbf{2}}\right)}, \boldsymbol{\Pi}^{\left(\mathbf{i}_{\mathbf{1}}, \mathbf{1}\right)}, \ldots, \boldsymbol{\Pi}^{\left(\mathbf{i}_{\mathbf{1}}, \mathbf{2} \mathbf{J}\right)}\right), \quad i_{1} \in V_{1}^{S} ; \\
\boldsymbol{\Pi}^{\left(\mathbf{0}, \mathbf{S}_{\mathbf{0}}\right)} & =\left(\pi^{\left(0, S_{0}, 0\right)}, \pi^{\left(0, S_{0}, 1\right)}, \ldots, \pi^{\left(0, S_{0}, N\right)}\right), \\
\boldsymbol{\Pi}^{\left(\mathbf{0}, \mathbf{i}_{\mathbf{2}}\right)} & =\left(\pi^{\left(0, i_{2}, 0\right)}, \pi^{\left(0, i_{2}, 1\right)}, \ldots, \pi^{\left(0, i_{2}, N-1\right)}\right), i_{2} \in V_{1}^{2 J} ; \\
\boldsymbol{\Pi}^{\left(\mathbf{i}_{1}, \mathbf{S}_{\mathbf{0}}\right)} & =\left(\pi^{\left(i_{1}, S_{0}, 0\right)}, \pi^{\left(i_{1}, S_{0}, 1\right)}, \ldots, \pi^{\left(i_{1}, S_{0}, N\right)}\right), i_{1} \in V_{1}^{S} ; \\
\boldsymbol{\Pi}^{\left(\mathbf{i}_{1}, \mathbf{S}_{\mathbf{1}}\right)} & =\left(\pi^{\left(i_{1}, S_{1}, 0\right)}, \pi^{\left(i_{1}, S_{1}, 1\right)}, \ldots, \pi^{\left(i_{1}, S_{1}, N-1\right)}\right), i_{1} \in V_{1}^{S} ; \\
\boldsymbol{\Pi}^{\left(\mathbf{i}_{1}, \mathbf{S}_{\mathbf{2}}\right)} & =\left(\pi^{\left(i_{1}, S_{2}, 0\right)}, \pi^{\left(i_{1}, S_{2}, 1\right)}, \ldots, \pi^{\left(i_{1}, S_{2}, N-1\right)}\right), i_{1} \in V_{1}^{S} ; \\
\boldsymbol{\Pi}^{\left(\mathbf{i}_{\mathbf{1}}, \mathbf{i}_{\mathbf{2}}\right)} & =\left(\pi^{\left(i_{1}, i_{2}, 0\right)}, \pi^{\left(i_{1}, i_{2}, 1\right)}, \ldots, \pi^{\left(i_{1}, i_{2}, N-1\right)}\right), i_{1} \in V_{1}^{S}, i_{2} \in V_{1}^{2 J} .
\end{aligned}
$$

Then, $\boldsymbol{\Pi}^{\left(\mathbf{i}_{1}\right)}$ is the probability vector $i_{1}$ th inventory level with each one element, stating a particular mixture of the inventory level and status of the server and the number of customers in the orbit. Using the vectors $\Pi^{\left(\mathbf{i}_{1}\right)}, i_{1} \in V_{0}^{S}$, the system of linear equations $(3.1)-(3.5)$ can be written as follows:

$$
\begin{aligned}
& \boldsymbol{\Pi}^{i_{1}} D_{i_{1}}+\boldsymbol{\Pi}^{i_{1}-1} C_{i_{1}-1}=\mathbf{0}, \quad i_{1}=1,2, \ldots, Q, \\
& \boldsymbol{\Pi}^{i_{1}} D_{i_{1}}+\boldsymbol{\Pi}^{\boldsymbol{i}_{1}-\mathbf{1}} C_{i_{1}-1}+\boldsymbol{\Pi}^{\left(i_{1}-\mathbf{1}-Q\right)} A_{1}=\mathbf{0}, \quad i_{1}=Q+1, \\
& \boldsymbol{\Pi}^{i_{1}} D_{i_{1}}+\boldsymbol{\Pi}^{i_{1}-1} C_{i_{1}-1}+\boldsymbol{\Pi}^{\left(i_{1}-1-Q\right)} A=\mathbf{0}, \quad i_{1}=Q+2, Q+3, \ldots, S, \\
& \Pi^{S} C_{S}+\Pi^{s} A=\mathbf{0} .
\end{aligned}
$$

Then, the limiting distribution vector $\boldsymbol{\Pi}=\left(\boldsymbol{\Pi}^{(\mathbf{0})}, \boldsymbol{\Pi}^{(\mathbf{1})}, \ldots, \boldsymbol{\Pi}^{(\mathbf{S})}\right)$ is the unique key of the system

$$
\Pi \Theta=\mathbf{0}
$$

and the normalization condition

$$
\boldsymbol{\Pi} \boldsymbol{e}=\sum \sum_{\left(i_{1}, i_{2}, i_{3}\right)} \sum \pi^{\left(i_{1}, i_{2}, i_{3}\right)}=1,
$$

where $\Theta=\left(\left(d\left(\left(i_{1}, i_{2}, i_{3}\right),\left(j_{1}, j_{2}, j_{3}\right)\right)\right)\right),\left(i_{1}, i_{2}, i_{3}\right),\left(j_{1}, j_{2}, j_{3}\right) \in E$ is the infinitesimal generator matrix of $\{a(t), t \geq 0\}$ can be expressed as follows:

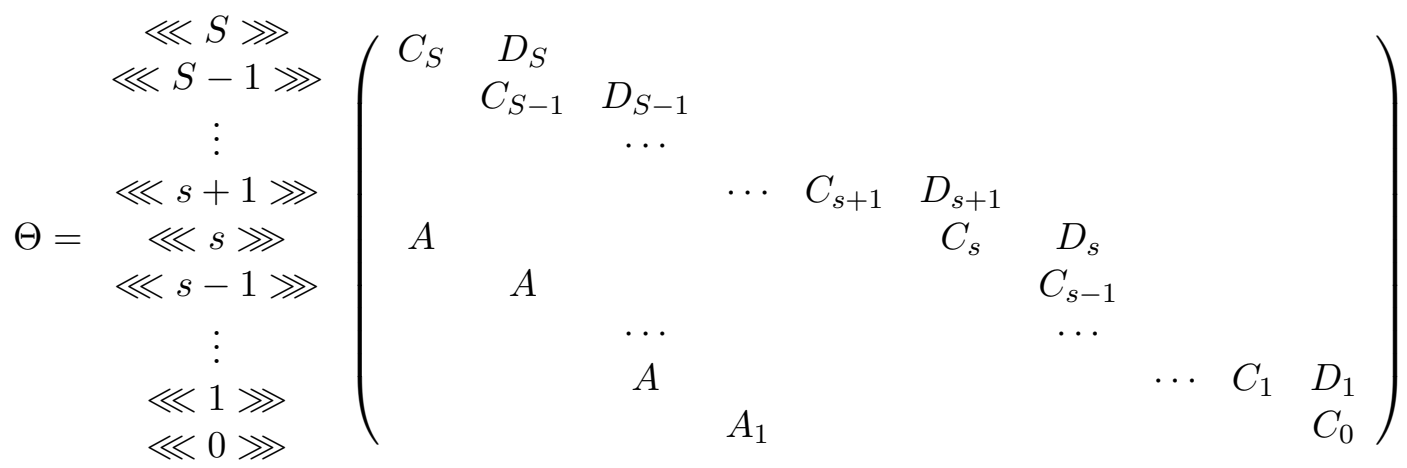


where $A_{1}=\left[a_{1\left(i_{1}, i_{2}, i_{3}\right),\left(j_{1}, j_{2}, j_{3}\right)}\right]$ is a rectangular matrix of size $(2 J+1) N+1 \times(2 J+3) N+1$ and its elements are given by

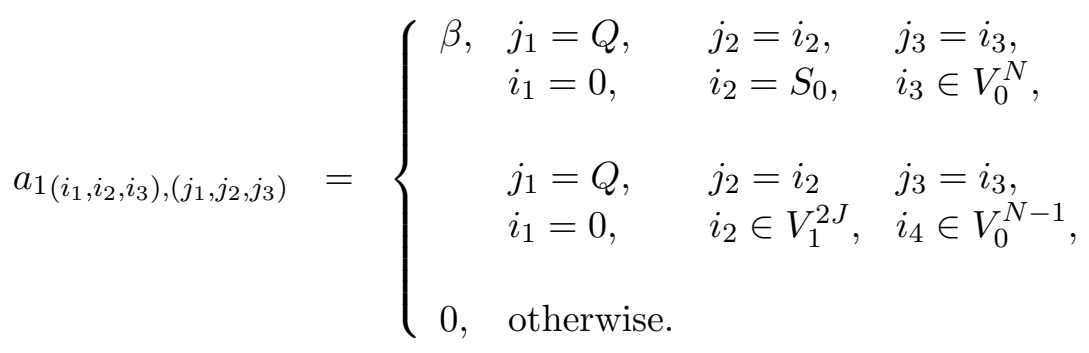

The matrix $A$ is a square matrix of size $((2 J+3) N+1)$ and it can be expressed as $A=\beta \times I_{((2 J+3) N+1)}$.

The matrices $D_{i_{1}}=\left[d^{i_{1}}{ }_{\left(i_{1}, i_{2}, i_{3}\right),\left(j_{1}, j_{2}, j_{3}\right)}\right], i_{1} \in V_{1}^{S}$ govern the transitions from the state $\lll i_{1} \ggg$ into the state $\lll i_{1}-1 \ggg$ and, hence $D_{1}$ is a rectangular matrix of size $(2 J+3) N+1 \times(2 J+1) N+1$ and other matrices $D_{i_{1}}, i_{1} \in V_{2}^{S}$ are square matrices of size $((2 J+3) N+1)$ with their the following elements, for $k \in V_{1}^{J}$

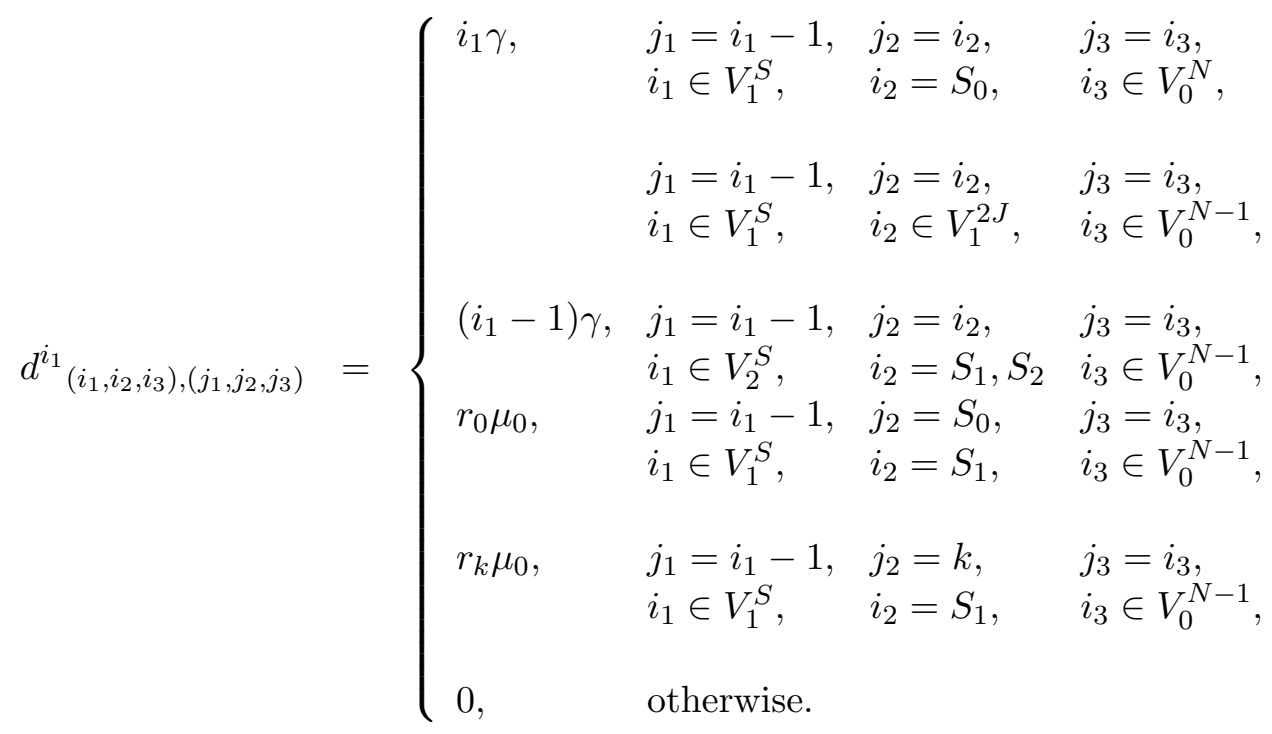

Finally, the matrices $C_{i_{1}}=\left[c^{i_{1}}{ }_{\left(i_{1}, i_{2}, i_{3}\right),\left(j_{1}, j_{2}, j_{3}\right)}\right], i_{1} \in V_{0}^{S}$ represents all transitions within $\lll i_{1} \ggg$ and $C_{0}$ is a square matrix of order $(N(2 J+1))+1$ and $C_{i_{1}}, i_{1} \in V_{1}^{S}$ are square matrices of order $(N(2 J+3))+1$ with the following elements, for $i_{1}=0, k \in V_{1}^{2 J}$

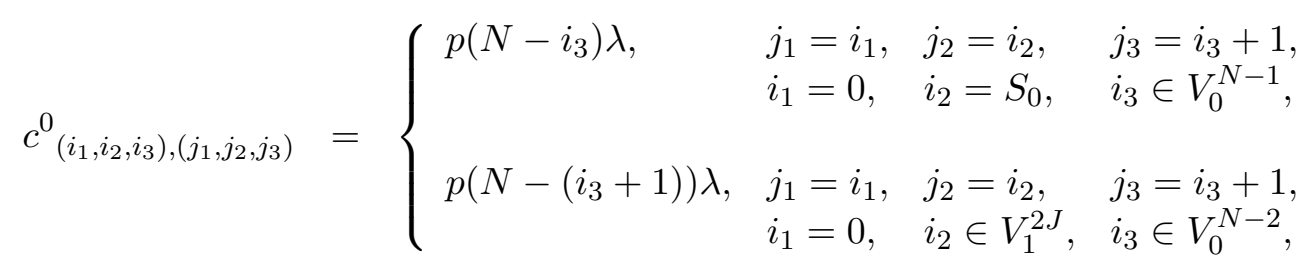




$$
\left\{\begin{array}{llll}
\mu_{i_{2}}, & j_{1}=i_{1}, & j_{2}=S_{0}, & j_{3}=i_{3}, \\
& i_{1}=0, & i_{2} \in V_{1}^{J} & i_{3} \in V_{0}^{N-1}, \\
\alpha_{i_{2}}, & j_{1}=i_{1}, & j_{2}=i_{2}+J, & j_{3}=i_{3}, \\
& i_{1}=0, & i_{2} \in V_{1}^{J}, & i_{3} \in V_{0}^{N-1}, \\
& j_{1}=i_{1}, & j_{2}=i_{2}-J, & j_{3}=i_{3}, \\
\eta_{i_{2}}, & i_{1}=0, & i_{2} \in V_{J+1}^{2 J}, & i_{3} \in V_{0}^{N-1}, \\
-h_{1}, & j_{1}=i_{1}, & j_{2}=i_{2}, & j_{3}=i_{3}, \\
& i_{1}=0, & i_{2}=S_{0}, & i_{3} \in V_{0}^{N}, \\
-h_{2}, & j_{1}=i_{1}, & j_{2}=i_{2}, & j_{3}=i_{3}, \\
& i_{1}=0, & i_{2} \in V_{1}^{J}, & i_{3} \in V_{0}^{N-1}, \\
-h_{3}, & j_{1}=i_{1}, & j_{2}=i_{2}, & \\
& i_{1}=0, & i_{2} \in V_{J+1}^{2 J}, & i_{3} \in i_{3}, \\
0, & &
\end{array}\right.
$$

where $h_{1}=\left(p\left(N-i_{3}\right) \lambda \bar{\delta}_{i_{3} N}+\beta\right), h_{2}=\left(p\left(N-\left(i_{3}+1\right)\right) \lambda \bar{\delta}_{i_{3}(N-1)}+\beta+\left(\alpha_{k}+\mu_{k}\right) \delta_{i_{2} k}\right), h_{3}=$ $\left(p\left(N-\left(i_{3}+1\right)\right) \lambda \bar{\delta}_{i_{3}(N-1)}+\beta+\eta(k-J) \delta_{i_{2} k}\right)$.

For $i_{1} \in V_{1}^{S}, k \in V_{1}^{2 J}$

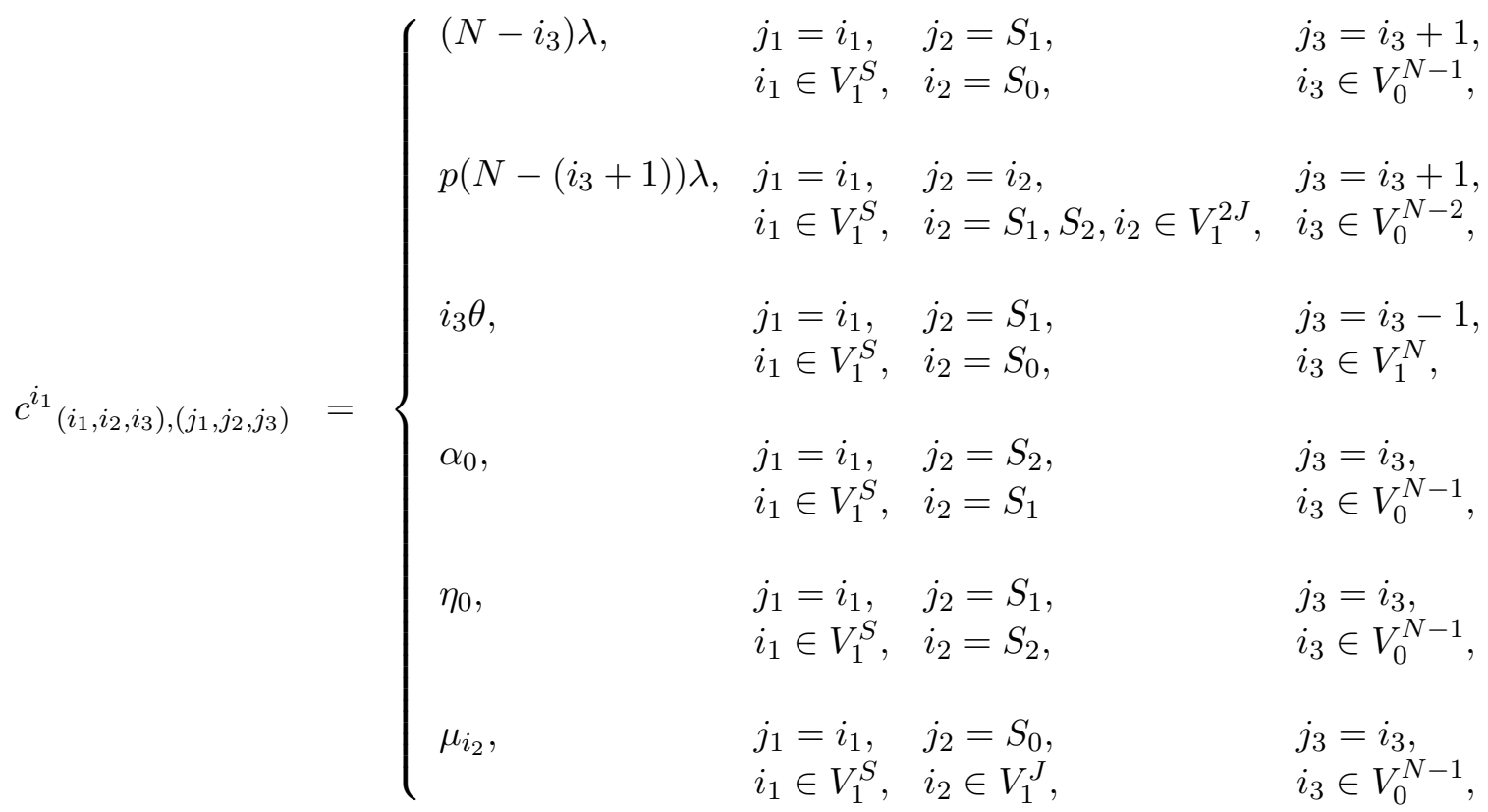




$$
\left\{\begin{array}{llll}
\alpha_{i_{2}}, & j_{1}=i_{1}, & j_{2}=i_{2}+J, & j_{3}=i_{3}, \\
& i_{1} \in V_{1}^{S}, & i_{2} \in V_{1}^{J}, & i_{3} \in V_{0}^{N-1}, \\
& \eta_{i_{2},}, i_{1}, & j_{2}=i_{2}-J, & j_{3}=i_{3}, \\
& i_{1} \in V_{1}^{S}, & i_{2} \in V_{J+1}^{2 J}, & i_{3} \in V_{0}^{N-1}, \\
-k_{1}, & j_{1}=i_{1}, & j_{2}=i_{2}, & j_{3}=i_{3}, \\
& i_{1} \in V_{1}^{S}, & i_{2}=S_{0}, & i_{3} \in V_{0}^{N}, \\
-k_{2}, & j_{1}=i_{1}, & j_{2}=i_{2}, & j_{3}=i_{3}, \\
& \in V_{1}^{S}, & i_{2}=S_{1}, S_{2}, & i_{3} \in V_{0}^{N-1}, \\
-k_{3}, & j_{1}=i_{1}, & j_{2}=i_{2}, & j_{3}=i_{3}, \\
& \in V_{1}^{S}, & i_{2} \in V_{1}^{J}, & i_{3} \in V_{0}^{N-1}, \\
-k_{4}, & j_{1}=i_{1}, & j_{2}=i_{2}, & j_{3}=i_{3}, \\
& \in V_{1}^{S}, & i_{2} \in V_{J+1}^{2 J}, & i_{3} \in V_{0}^{N-1}, \\
0, & &
\end{array}\right.
$$

where $k_{1}=\left(\left(N-i_{3}\right) \lambda \bar{\delta}_{i_{3} N}+i_{1} \gamma+H\left(s-i_{1}\right) \beta+i_{3} \theta\right), k_{2}=\left(p\left(N-\left(i_{3}+1\right)\right) \lambda \bar{\delta}_{i_{3}(N-1)}+\right.$ $\left.H\left(s-i_{1}\right) \beta+\left(i_{1}-1\right) \gamma+\left(\alpha_{0}+\mu_{0}\right) \delta_{i_{2} S_{1}}+\delta_{i_{2} S_{2}} \eta_{0}\right), k_{3}=\left(p\left(N-\left(i_{3}+1\right)\right) \lambda \bar{\delta}_{i_{3}(N-1)}+H(s-\right.$ $\left.\left.i_{1}\right) \beta+i_{1} \gamma+\left(\alpha_{k}+\mu_{k}\right) \delta_{i_{2} k}\right), k_{4}=\left(p\left(N-\left(i_{3}+1\right)\right) \lambda \bar{\delta}_{i_{3}(N-1)}+H\left(s-i_{1}\right) \beta+i_{1} \gamma+\eta_{(k-J)} \delta_{i_{2} k}\right)$.

A recursive algorithm is now derived for the solutions of the steady-state equations (3.6) and (3.7). The steady state probability vector $\boldsymbol{\Pi}^{\left(\boldsymbol{i}_{1}\right)}, i_{1} \in V_{0}^{S}$ can be determined from an algorithm given by the following steps.

Step 1. To obtain the value of $\Pi^{Q}$, the following system of equations is described:

$$
\begin{aligned}
& \boldsymbol{\Pi}^{Q}\left[\left\{(-1)^{Q} \sum_{j=0}^{s-1}\left[\left(\underset{k=Q}{s+1-j} D_{k} C_{k-1}^{-1}\right) A C_{S-j}^{-1}\left(\underset{l=S-j}{\Omega+2} D_{l} C_{l-1}^{-1}\right)\right]\right\} D_{Q+1}\right. \\
& \left.+C_{Q}+\left\{(-1)^{Q} \underset{j=Q}{\stackrel{1}{\Omega}} D_{j} C_{j-1}^{-1}\right\} A\right]=\mathbf{0},
\end{aligned}
$$

and

$$
\begin{aligned}
& \boldsymbol{\Pi}^{Q}\left[\sum_{i_{1}=0}^{Q-1}\left((-1)^{Q-i_{1}} \underset{j=Q}{i_{1}+1} D_{j} C_{j-1}^{-1}\right)+I\right. \\
& \left.+\sum_{i_{1}=Q+1}^{S}\left((-1)^{2 Q-i_{1}+1} \sum_{j=0}^{S-i_{1}}\left[\left(\underset{k=Q}{s+1-j} D_{k} C_{k-1}^{-1}\right) A C_{S-j}^{-1}\left(\underset{l=S-j}{i_{1}+1} D_{l} C_{l-1}^{-1}\right)\right]\right)\right] \boldsymbol{e}=1 .
\end{aligned}
$$

Step 2. Next, the following values are computed by 


$$
\begin{aligned}
& \boldsymbol{\Omega}_{\boldsymbol{i}_{\mathbf{1}}}=(-1)^{Q-i_{1}} \boldsymbol{\Pi}^{\boldsymbol{Q}^{i_{1}+1}} \underset{j=Q}{\Omega} D_{j} C_{j-1}^{-1}, \quad i_{1}=Q-1, Q-2, \ldots, 0 \\
& =(-1)^{2 Q-i_{1}+1} \Pi^{Q} \sum_{j=0}^{S-i_{1}}\left[\left(\underset{k=Q}{s+1-j} D_{k} C_{k-1}^{-1}\right) A C_{S-j}^{-1}\left(\underset{l=S-j}{\Omega_{1}+1} D_{l} C_{l-1}^{-1}\right)\right], \\
& =I, \quad i_{1}=Q \text {. } \\
& i_{1}=S, S-1, \ldots, Q+1
\end{aligned}
$$

Step 3. By $\boldsymbol{\Pi}^{(\mathbf{Q})}$ and $\Omega_{i_{1}}, i_{1}=0,1, \ldots, S$, get the value of $\boldsymbol{\Pi}^{\left(\mathbf{i}_{1}\right)}, i_{1}=0,1, \ldots, S$. Explicitly,

$$
\boldsymbol{\Pi}^{\left(\mathbf{i}_{1}\right)}=\boldsymbol{\Pi}^{(\mathbf{Q})} \Omega_{i_{1}}, \quad i_{1}=0,1, \ldots, S
$$

\section{Waiting time analysis of an orbital customer}

In this section, the waiting time distribution of an orbital customer is discussed that is specified as the time between the arrival times of the customer and moment upon which he/she gets service. This continuous time random variable is symbolized as $W$. The objective is to calculate the probability distribution of $W$ and to calculate $n^{\text {th }}$ order moments of $W$. If the arriving customer finds the system in state $\left(i_{1}, S_{0}, i_{3}\right), i_{1} \in V_{1}^{S}, i_{3} \in$ $V_{0}^{N-1}$, he/she gets the service immediately. Therefore, $W=0$. The probability for the customer does not wait is denoted by $P\{W=0\}$ and is given by,

$$
P\{W=0\}=\sum_{i_{1}=1}^{S} \sum_{i_{3}=0}^{N-1} \pi^{\left(i_{1}, S_{0}, 0\right)} .
$$

To obtain the distribution of $W$, some auxiliary variables are defined. Assume that the system is in the state $\left(i_{1}, i_{2}, i_{3}\right), i_{3}>0$ at an arbitrary time $t$. Let $W^{*}(y)=E\left[e^{-y W}\right]$ be the Laplace-Stieltjes transform of the unconditional waiting time and let $W_{\left(i_{1}, i_{2}, i_{3}\right)}^{*}(y)=$ $E\left[e^{-y W_{\left(i_{1}, i_{2}, i_{3}\right)}}\right]$ be the Laplace-Stieltjes transform of the conditional waiting time. Thus,

$$
\begin{aligned}
W^{*}(y) & =\sum_{i_{1}=1}^{S} \sum_{i_{3}=0}^{N-1} \pi^{\left(i_{1}, S_{0}, i_{3}\right)}+\sum_{i_{3}=0}^{N-1} \pi^{\left(0, S_{0}, i_{3}\right)} W_{\left(0, S_{0}, i_{3}+1\right)}^{*}(y)+\sum_{i_{1}=1}^{S} \sum_{i_{3}=0}^{N-2} \pi^{\left(i_{1}, S_{1}, i_{3}\right)} W_{\left(i_{1}, S_{1}, i_{3}+1\right)}^{*}(y) \\
& +\sum_{i_{1}=1}^{S} \sum_{i_{3}=0}^{N-2} \pi^{\left(i_{1}, S_{2}, i_{3}\right)} W_{\left(i_{1}, S_{2}, i_{3}+1\right)}^{*}(y)+\sum_{i_{1}=0}^{S} \sum_{i_{2}=1}^{2 J} \sum_{i_{3}=0}^{N-2} \pi^{\left(i_{1}, i_{2}, i_{3}\right)} W_{\left(i_{1}, i_{2}, i_{3}+1\right)}^{*}(y) .
\end{aligned}
$$

To derive $W_{\left(i_{1}, i_{2}, i_{3}\right)}^{*}$, an auxiliary Markov chain is introduced on the state space

$$
\begin{aligned}
E^{*}= & \{*\} \cup\left\{0, i_{2}=S_{0}, 0 \leq i_{3} \leq N\right\} \cup \\
& \left\{1 \leq i_{1} \leq S, i_{2}=S_{1}, S_{2}, 0 \leq i_{3} \leq N-1\right\} \cup \\
& \left\{0 \leq i_{1} \leq S, 1 \leq i_{2} \leq 2 J, 0 \leq i_{3} \leq N-1\right\},
\end{aligned}
$$


where $\{*\}$ represents an absorbing state. The chain is on a state $\left(i_{1}, i_{2}, i_{3}\right)$, a first-step argument is applied in the auxiliary chain to resolve $W_{\left(i_{1}, i_{2}, i_{3}\right)}^{*}(y)$. Then (see [14], Theorem 6.21$)$ the functions $W_{\left(i_{1}, i_{2}, i_{3}\right)}^{*}(y),\left(i_{1}, i_{2}, i_{3}\right) \in E^{*}$ are the least non-negative solution to the system.

For $0 \leq i_{1} \leq S, \quad i_{2}=S_{0}, \quad 1 \leq i_{3} \leq N$

$$
\begin{array}{r}
w_{1} W_{\left(i_{1}, i_{2}, i_{3}\right)}^{*}(y)=p\left(N-i_{3}\right) \lambda \bar{\delta}_{i_{3} N} \delta_{i_{1} 0} W_{\left(i_{1}, S_{0}, i_{3}+1\right)}^{*}(y)+\left(N-i_{3}\right) \lambda \bar{\delta}_{i_{3} N} \bar{\delta}_{i_{1} 0} W_{\left(i_{1}, S_{1}, i_{3}\right)}^{*}(y) \\
+i_{1} \gamma \bar{\delta}_{i_{1} 0} W_{\left(i_{1}-1, S_{0}, i_{3}\right)}^{*}(y)+\beta H\left(s-i_{1}\right) W_{\left(i_{1}+Q, S_{0}, i_{3}\right)}^{*}(y)+i_{3} \theta \bar{\delta}_{i_{1} 0} W_{\left(i_{1}, S_{1}, i_{3}-1\right)}^{*}(y)
\end{array}
$$

where

$$
w_{1}=y+p\left(N-i_{3}\right) \lambda \bar{\delta}_{i_{3} N} \delta_{i_{1} 0}+\left(N-i_{3}\right) \lambda \bar{\delta}_{i_{3} N} \bar{\delta}_{i_{1} 0}+i_{1} \gamma \bar{\delta}_{i_{1} 0}+\beta H\left(s-i_{1}\right)+i_{3} \theta \bar{\delta}_{i_{1} 0}
$$

For $1 \leq i_{1} \leq S, \quad i_{2}=S_{1}, S_{2} \quad 1 \leq i_{3} \leq N-1$

$$
\begin{array}{r}
w_{2} W_{\left(i_{1}, i_{2}, i_{3}\right)}^{*}(y)=p\left(N-\left(i_{3}+1\right)\right) \lambda \bar{\delta}_{i_{3}(N-1)} W_{\left(i_{1}, i_{2}, i_{3}+1\right)}^{*}(y)+\bar{\delta}_{i_{1} 1}\left(i_{1}-1\right) \gamma W_{\left(i_{1}-1, i_{2}, i_{3}\right)}^{*}(y)(10) \\
+\beta H\left(s-i_{1}\right) W_{\left(i_{1}+Q, i_{2}, i_{3}\right)}^{*}(y)+\delta_{i_{2} S_{1}} \alpha_{0} W_{\left(i_{1}, S_{2}, i_{3}\right)}^{*}(y)+\delta_{i_{2} S_{2}} \eta_{0} W_{\left(i_{1}, S_{1}, i_{3}\right)}^{*}(y) \\
+\sum_{k=1}^{J}\left(\delta_{i_{2} S_{1}} r_{k} \mu_{0}\right) W_{\left(i_{1}-1, k, i_{3}\right)}^{*}(y)
\end{array}
$$

where

$$
\begin{aligned}
w_{2}= & y+p\left(N-\left(i_{3}+1\right)\right) \lambda \bar{\delta}_{i_{3}(N-1)}+\bar{\delta}_{i_{1} 1}\left(i_{1}-1\right) \gamma+\beta H\left(s-i_{1}\right)+\delta_{i_{2} S_{1}} \alpha_{0} \\
& +\delta_{i_{2} S_{2}} \eta_{0}+\sum_{k=1}^{J}\left(\delta_{i_{2} S_{1}} r_{k} \mu_{0}\right) .
\end{aligned}
$$

For $0 \leq i_{1} \leq S, \quad 1 \leq i_{2} \leq J, \quad 1 \leq i_{3} \leq N-1$

$$
\begin{aligned}
& w_{3} W_{\left(i_{1}, i_{2}, i_{3}\right)}^{*}(y)=p\left(N-\left(i_{3}+1\right)\right) \lambda \bar{\delta}_{i_{3}(N-1)} W_{\left(i_{1}, i_{2}, i_{3}+1\right)}^{*}(y)+\bar{\delta}_{i_{1} 0} i_{1} \gamma W_{\left(i_{1}-1, i_{2}, i_{3}\right)}^{*}(y) \\
& +\beta H\left(s-i_{1}\right) W_{\left(i_{1}+Q, i_{2}, i_{3}\right)}^{*}(y)+\sum_{k=1}^{J}\left(\delta_{i_{2} k} \mu_{k}\right) W_{\left(i_{1}, S_{0}, i_{3}\right)}^{*}(y)+\sum_{k=1}^{J}\left(\delta_{i_{2} k} \alpha_{k}\right) W_{\left(i_{1}, k, i_{3}\right)}^{*}(y)
\end{aligned}
$$

where

$$
w_{3}=y+p\left(N-\left(i_{3}+1\right)\right) \lambda \bar{\delta}_{i_{3}(N-1)}+\bar{\delta}_{i_{1} 0} i_{1} \gamma+\beta H\left(s-i_{1}\right)+\sum_{k=1}^{J}\left(\delta_{i_{2} k} \mu_{k}\right)+\sum_{k=1}^{J}\left(\delta_{i_{2} k} \alpha_{k}\right) .
$$

For $0 \leq i_{1} \leq S, \quad J+1 \leq i_{2} \leq 2 J, \quad 1 \leq i_{3} \leq N-1$,

$$
\begin{aligned}
w_{4} W_{\left(i_{1}, i_{2}, i_{3}\right)}^{*}(y)=p(N- & \left.\left(i_{3}+1\right)\right) \lambda \bar{\delta}_{i_{3}(N-1)} W_{\left(i_{1}, i_{2}, i_{3}+1\right)}^{*}(y)+\bar{\delta}_{i_{1} 0} i_{1} \gamma W_{\left(i_{1}-1, i_{2}, i_{3}\right)}^{*}(y) \\
& +\beta H\left(s-i_{1}\right) W_{\left(i_{1}+Q, i_{2}, i_{3}\right)}^{*}(y)+\sum_{k=1}^{J}\left(\delta_{i_{2}(J+k)} \eta_{k}\right) W_{\left(i_{1}, k, i_{3}\right)}^{*}(y)
\end{aligned}
$$


where

$$
w_{4}=y+p\left(N-\left(i_{3}+1\right)\right) \lambda \bar{\delta}_{i_{3}(N-1)}+\bar{\delta}_{i_{1} 0} i_{1} \gamma+\beta H\left(s-i_{1}\right)+\sum_{k=1}^{J}\left(\delta_{i_{2}(J+k)} \eta_{k}\right) .
$$

The system of linear equations can be utilized to obtain a recursive algorithm for calculating the moments for the waiting times. By differentiating the system of linear equations (4.2)-(4.5) a total of $(n+1)$ times and evaluating at $y=0$, the following is obtained.

For $0 \leq i_{1} \leq S, \quad i_{2}=S_{0}, \quad 1 \leq i_{3} \leq N$,

$$
\begin{array}{r}
w_{5} E\left[W_{\left(i_{1}, i_{2}, i_{3}\right)}^{(n+1)}\right]-p\left(N-i_{3}\right) \lambda \bar{\delta}_{i_{3} N} \delta_{i_{1} 0} E\left[W_{\left(i_{1}, S_{0}, i_{3}+1\right)}^{(n+1)}\right]-\left(N-i_{3}\right) \lambda \bar{\delta}_{i_{3} N} \bar{\delta}_{i_{1} 0} E\left[W_{\left(i_{1}, S_{1}, i_{3}\right)}^{(n+1)}\right] \\
-i_{1} \gamma \bar{\delta}_{i_{1} 0} E\left[W_{\left(i_{1}-1, S_{0}, i_{3}\right)}^{(n+1)}\right]-\beta H\left(s-i_{1}\right) E\left[W_{\left(i_{1}+Q, S_{0}, i_{3}\right)}^{(n+1)}\right]-\left(i_{3}-1\right) \theta \bar{\delta}_{i_{1} 0} E\left[W_{\left(i_{1}, S_{1}, i_{3}-1\right)}^{(n+1)}\right] \\
=(n+1) E\left[W_{\left(i_{1}, i_{2}, i_{3}\right)}^{(n)}\right]
\end{array}
$$

where

$$
w_{5}=p\left(N-i_{3}\right) \lambda \bar{\delta}_{i_{3} N} \delta_{i_{1} 0}+\left(N-i_{3}\right) \lambda \bar{\delta}_{i_{3} N} \bar{\delta}_{i_{1} 0}+i_{1} \gamma \bar{\delta}_{i_{1} 0}+\beta H\left(s-i_{1}\right)+i_{3} \theta \bar{\delta}_{i_{1} 0} .
$$

For $1 \leq i_{1} \leq S, \quad i_{2}=S_{1}, S_{2}, \quad 1 \leq i_{3} \leq N-1$,

$$
\begin{array}{r}
w_{6} E\left[W_{\left(i_{1}, i_{2}, i_{3}\right)}^{(n+1)}\right]-p\left(N-\left(i_{3}+1\right)\right) \lambda \bar{\delta}_{i_{3}(N-1)} E\left[W_{\left(i_{1}, i_{2}, i_{3}+1\right)}^{(n+1)}\right]-\bar{\delta}_{i_{1} 1}\left(i_{1}-1\right) \gamma E\left[W_{\left(i_{1}-1, i_{2}, i_{3}\right)}^{(n+1)}\right] \\
-\beta H\left(s-i_{1}\right) E\left[W_{\left(i_{1}+Q, i_{2}, i_{3}\right)}^{(n+1)}\right]-\delta_{i_{2} S_{1}} \alpha_{0} E\left[W_{\left(i_{1}, S_{2}, i_{3}\right)}^{(n+1)}\right]-\delta_{i_{2} S_{2}} \eta_{0} E\left[W_{\left(i_{1}, S_{1}, i_{3}\right)}^{(n+1)}\right] \\
-\sum_{k=1}^{J}\left(\delta_{i_{2} S_{1}} r_{k} \mu_{0}\right) E\left[W_{\left(i_{1}-1, k, i_{3}\right)}^{(n+1)}\right]=(n+1) E\left[W_{\left(i_{1}, i_{2}, i_{3}\right)}^{(n)}\right]
\end{array}
$$

where

$$
\begin{aligned}
w_{6}= & p\left(N-\left(i_{3}+1\right)\right) \lambda \bar{\delta}_{i_{3}(N-1)}+\bar{\delta}_{i_{1} 1}\left(i_{1}-1\right) \gamma+\beta H\left(s-i_{1}\right)+\delta_{i_{2} S_{1}} \alpha_{0} \\
& +\delta_{i_{2} S_{2}} \eta_{0}+\sum_{k=1}^{J}\left(\delta_{i_{2} S_{1}} r_{k} \mu_{0}\right) .
\end{aligned}
$$

For $0 \leq i_{1} \leq S, \quad 1 \leq i_{2} \leq J, \quad 1 \leq i_{3} \leq N-1$,

$$
\begin{array}{r}
w_{7} E\left[W_{\left(i_{1}, i_{2}, i_{3}\right)}^{(n+1)}\right]-p\left(N-\left(i_{3}+1\right)\right) \lambda \bar{\delta}_{i_{3}(N-1)} E\left[W_{\left(i_{1}, i_{2}, i_{3}+1\right)}^{(n+1)}\right]-\bar{\delta}_{i_{1} 0} i_{1} \gamma E\left[W_{\left(i_{1}-1, i_{2}, i_{3}\right)}^{(n+1)}\right] \\
-\beta H\left(s-i_{1}\right) E\left[W_{\left(i_{1}+Q, i_{2}, i_{3}\right)}^{(n+1)}\right]-\sum_{k=1}^{J}\left(\delta_{i_{2} k} \mu_{k}\right) E\left[W_{\left(i_{1}, S_{0}, i_{3}\right)}^{(n+1)}\right]-\sum_{k=1}^{J}\left(\delta_{i_{2} k} \alpha_{k}\right) E\left[W_{\left(i_{1}, k, i_{3}\right)}^{(n+1)}\right] \\
=(n+1) E\left[W_{\left(i_{1}, i_{2}, i_{3}\right)}^{(n)}\right]
\end{array}
$$

where

$$
w_{7}=p\left(N-\left(i_{3}+1\right)\right) \lambda \bar{\delta}_{i_{3}(N-1)}+\bar{\delta}_{i_{1} 0} i_{1} \gamma+\beta H\left(s-i_{1}\right)+\sum_{k=1}^{J}\left(\delta_{i_{2} k} \mu_{k}\right)+\sum_{k=1}^{J}\left(\delta_{i_{2} k} \alpha_{k}\right) .
$$


For $0 \leq i_{1} \leq S, \quad J+1 \leq i_{2} \leq 2 J, \quad 1 \leq i_{3} \leq N-1$,

$$
\begin{aligned}
& w_{8} E\left[W_{\left(i_{1}, i_{2}, i_{3}\right)}^{(n+1)}\right]-p\left(N-\left(i_{3}+1\right)\right) \lambda \bar{\delta}_{i_{3}(N-1)} E\left[W_{\left(i_{1}, i_{2}, i_{3}+1\right)}^{(n+1)}\right]-i_{1} \gamma \bar{\delta}_{i_{1} 0} E\left[W_{\left(i_{1}-1, i_{2}, i_{3}\right)}^{(n+1)}\right] \\
& -\beta H\left(s-i_{1}\right) E\left[W_{\left(i_{1}+Q, i_{2}, i_{3}\right)}^{(n+1)}\right]-\sum_{k=1}^{J}\left(\delta_{i_{2}(J+k)} \eta_{k}\right) E\left[{ }^{(1)} W_{\left(i_{1}, k, i_{3}\right)}^{(n+1)}\right]=(n+1) E\left[W_{\left(i_{1}, i_{2}, i_{3}\right)}^{(n)}\right]
\end{aligned}
$$

where

$$
w_{8}=p\left(N-\left(i_{3}+1\right)\right) \lambda \bar{\delta}_{i_{3}(N-1)}+\bar{\delta}_{i_{1} 0} i_{1} \gamma+\beta H\left(s-i_{1}\right)+\sum_{k=1}^{J}\left(\delta_{i_{2}(J+k)} \eta_{k}\right)
$$

Using the linear equations (4.6)-(4.9), the unknowns $E\left[W_{\left(i_{1}, i_{2}, i_{3}\right)}^{(n+1)}\right],\left(i_{1}, i_{2}, i_{3}\right) \in E \operatorname{can}$ be determined in terms of one order less. Since $E\left[W_{\left(i_{1}, i_{2}, i_{3},\right)}^{(n)}\right]=1$, when $n=0$. The moments can be obtained up to a required order in a recursive way.

That is, for $n \geq 0$,

$$
\begin{aligned}
& E\left[W^{(n)}\right]=\delta_{0 n}+\left[\sum_{1_{3}=0}^{N-1} \phi^{\left(0, S_{0}, i_{3}\right)} E\left[W_{\left(0, S_{0}, i_{3}+1\right)}^{(n)}\right]+\sum_{i_{1}=1}^{S} \sum_{i_{3}=0}^{N-2} \phi^{\left(i_{1}, S_{1}, i_{3}+1\right)} E\left[W_{\left(i_{1}, S_{1}, i_{3}+1\right)}^{(n)}\right]\right. \\
& \left.+\sum_{i_{1}=1}^{S} \sum_{i_{3}=0}^{N-2} \phi^{\left(i_{1}, S_{2}, i_{3}\right)} E\left[W_{\left(i_{1}, S_{2}, i_{3}+1\right)}^{(n)}\right]+\sum_{i_{1}=0}^{S} \sum_{i_{2}=1}^{2 J} \sum_{i_{3}=0}^{N-2} \phi^{\left(i_{1}, i_{2}, i_{3}\right)} E\left[W_{\left(i_{1}, i_{2}, i_{3}+1\right)}^{(n)}\right]\right]\left(1-\delta_{0 n}\right),
\end{aligned}
$$

which provides the $n^{\text {th }}$ moments of the unconditional waiting time in terms of conditional moments of the same order.

\section{$5 \quad$ System performance measures}

In this section, some measures of system performance are derived in the steady state. Using this, the total expected cost rate is calculated.

\subsection{Average on-hand inventory level}

Let $\eta_{I}$ denote the average on-hand inventory level in the steady state. Then

$$
\eta_{I}=\sum_{i_{1}=1}^{S} i_{1} \Pi^{\left(i_{1}\right)} \mathbf{e}
$$

\subsection{Mean reorder rate}

Let $\eta_{R}$ denote the mean reorder rate in the steady state. A reorder is placed when the inventory level drops from $s+1$ to $s$. This may occur in the following cases: 
- The server completes the essential service of a customer,

- any one of the $(s+1)$ items fails when the server is idle,

- any one of the $s$ items fails either when the server is busy with FES or when the server is on interruption during FES providing,

- any one of the $(s+1)$ items fails either when the server is busy with Type $k(1 \leq$ $k \leq J)$ service or when server is on interruption during Type $k$ service providing.

Hence,

$$
\begin{aligned}
\eta_{R}=\sum_{i_{3}=0}^{N-1}\left\{\left(\sum_{k=0}^{J} r_{k} \mu_{0}\right)+s \gamma\right\} \pi^{\left(s+1, S_{1}, i_{3}\right)}+\sum_{i_{3}=0}^{N}(s+1) \gamma \pi^{\left(s+1, S_{0}, i_{3}\right)} \\
+\sum_{i_{3}=0}^{N-1} s \gamma \pi^{\left(s+1, S_{2}, i_{3}\right)}+\sum_{i_{2}=1}^{2 J} \sum_{i_{3}=0}^{N-1}(s+1) \gamma \pi^{\left(s+1, i_{2}, i_{3}\right)}
\end{aligned}
$$

\subsection{Mean perishable rate}

Let $\eta_{P}$ denote the mean perishable rate in the steady state. Then

$$
\begin{array}{r}
\eta_{P}=\sum_{i_{1}=1}^{S} \sum_{i_{3}=0}^{N} i_{1} \gamma \pi^{\left(i_{1}, S_{0}, i_{3}\right)}+\sum_{i_{1}=1}^{S} \sum_{i=1}^{2} \sum_{i_{3}=0}^{N-1}\left(i_{1}-1\right) \gamma \pi^{\left(i_{1}, S_{i}, i_{3}\right)} \\
+\sum_{i_{1}=1}^{S} \sum_{i_{2}=1}^{2 J} \sum_{i_{3}=0}^{N-1} i_{1} \gamma \pi^{\left(i_{1}, i_{2}, i_{3}\right)}
\end{array}
$$

\subsection{Average number of customers in the orbit}

Let $\eta_{C O}$ denote the average number of customers in the steady state. Then

$$
\eta_{C O}=\sum_{i_{1}=0}^{S} \sum_{i_{3}=1}^{N} i_{3} \pi^{\left(i_{1}, S_{0}, i_{3}\right)}+\sum_{i_{1}=1}^{S} \sum_{i=1}^{2} \sum_{i_{3}=1}^{N-1} i_{3} \pi^{\left(i_{1}, S_{i}, i_{3}\right)}+\sum_{i_{1}=0}^{S} \sum_{i_{2}=1}^{2 J} \sum_{i_{3}=1}^{N-1} i_{3} \pi^{\left(i_{1}, i_{2}, i_{3}\right)}
$$

\subsection{Average number of customers lost to the system}

Let $\eta_{C L}$ denote the average number of customers lost to the system in the steady state. Then

$$
\begin{aligned}
\eta_{C L}= & \sum_{i_{3}=0}^{N-1}(1-p)\left(N-i_{3}\right) \lambda \pi^{\left(0, S_{0}, i_{3}\right)}+\sum_{i_{1}=1}^{S} \sum_{i=1}^{2} \sum_{i_{3}=0}^{N-2}(1-p)\left(N-\left(i_{3}+1\right)\right) \lambda \pi^{\left(i_{1}, S_{i}, i_{3}\right)}+ \\
& \sum_{i_{1}=0}^{S} \sum_{i_{2}=1}^{2 J} \sum_{i_{3}=0}^{N-2}(1-p)\left(N-\left(i_{3}+1\right)\right) \lambda \pi^{\left(i_{1}, i_{2}, i_{3}\right)} .
\end{aligned}
$$




\subsection{Effective interruption rate}

Let $\eta_{I N T R}$ denote the effective interruption rate in the steady state. Then

$$
\eta_{I N T R}=\sum_{i_{1}=1}^{S} \sum_{i_{3}=0}^{N-1} \alpha_{0} \pi^{\left(i_{1}, S_{1}, i_{3}\right)}+\sum_{i_{1}=0}^{S} \sum_{i_{2}=1}^{J} \sum_{i_{3}=0}^{N-1} \alpha_{i_{2}} \pi^{\left(i_{1}, i_{2}, i_{3}\right)} .
$$

\subsection{Effective repair rate}

Let $\eta_{R I}$ denote the effective repair rate in the steady state. Then

$$
\eta_{R I}=\sum_{i_{1}=1}^{S} \sum_{i_{3}=0}^{N-1} \eta_{0} \pi^{\left(i_{1}, S_{2}, i_{3}\right)}+\sum_{i_{1}=0}^{S} \sum_{i_{2}=J+1}^{2 J} \sum_{i_{3}=0}^{N-1} \eta_{\left(i_{2}-J\right)} \pi^{\left(i_{1}, i_{2}, i_{3}\right)} .
$$

\subsection{The overall retrial rate}

Let $\eta_{O R}$ denote the overall rate of retrials in the steady state. Then

$$
\eta_{O R}=\sum_{i_{1}=0}^{S} \sum_{i_{3}=1}^{N} i_{3} \theta \pi^{\left(i_{1}, S_{0}, i_{3}\right)}+\sum_{i_{1}=1}^{S} \sum_{i=1}^{2} \sum_{i_{3}=1}^{N-1} i_{3} \theta \pi^{\left(i_{1}, S_{i}, i_{3}\right)}+\sum_{i_{1}=0}^{S} \sum_{i_{2}=1}^{2 J} \sum_{i_{3}=1}^{N-1} i_{3} \theta \pi^{\left(i_{1}, i_{2}, i_{3}\right)} .
$$

\subsection{The successful retrial rate}

Let $\eta_{S R}$ denote the successful retrial rate in the steady state. Then

$$
\eta_{S R}=\sum_{i_{1}=1}^{S} \sum_{i_{3}=1}^{N} i_{3} \theta \pi^{\left(i_{1}, S_{0}, i_{3}\right)}
$$

\subsection{The fraction of successful rate of retrial}

Let $\eta_{F R}$ denote the fraction of successful retrial rate in the steady state. Then

$$
\eta_{F R}=\frac{\eta_{S R}}{\eta_{O R}} .
$$

\subsection{The probability that the server and system are idle}

Let $\eta_{P S I}$ denote the probability that server and system are idle is given by

$$
\eta_{P S I}=\sum_{i_{1}=0}^{S} \pi^{\left(i_{1}, S_{0}, 0\right)} \text {. }
$$

\subsection{The probability that the server is idle, but the system is not empty}

Let $\eta_{P S N I}$ denote the probability that server is idle and system is not empty is given by

$$
\eta_{P S N I}=\sum_{i_{1}=0}^{S} \sum_{i_{3}=1}^{N} \pi^{\left(i_{1}, S_{0}, i_{3}\right)} \text {. }
$$




\subsection{Probability that the server is busy (FES or SOS)}

Let $\eta_{P S B}$ denote the probability that server is busy is given by

$$
\eta_{P S B}=\sum_{i_{1}=1}^{S} \sum_{i_{3}=0}^{N-1} \pi^{\left(i_{1}, S_{1}, i_{3}\right)}+\sum_{i_{1}=0}^{S} \sum_{i_{2}=1}^{J} \sum_{i_{3}=0}^{N-1} \pi^{\left(i_{1}, i_{2}, i_{3}\right)}
$$

\subsection{Probability that the server is under repair}

Let $\eta_{P O R}$ denote the probability that server is under repair is given by

$$
\eta_{P O R}=\sum_{i_{1}=1}^{S} \sum_{i_{3}=0}^{N-1} \pi^{\left(i_{1}, S_{2}, i_{3}\right)}+\sum_{i_{1}=0}^{S} \sum_{i_{2}=J+1}^{2 J} \sum_{i_{3}=0}^{N-1} \pi^{\left(i_{1}, i_{2}, i_{3}\right)} .
$$

\subsection{The effective rate at which arriving customers are lost on seeing an interrupted server}

Let $\eta_{P O R}$ denote the effective rate at which arriving customers are lost on seeing an interrupted server is given by

$$
\begin{aligned}
\eta_{L I}= & \sum_{i_{1}=1}^{S} \sum_{i_{3}=0}^{N-2}(1-p)\left(N-\left(i_{3}+1\right)\right) \lambda \pi^{\left(i_{1}, S_{2}, i_{3}\right)}+\sum_{i_{1}=0}^{S} \sum_{i_{2}=J+1}^{2 J} \sum_{i_{3}=0}^{N-2}(1-p)(N- \\
& \left.\left(i_{3}+1\right)\right) \lambda \pi^{\left(i_{1}, i_{2}, i_{3}\right)} .
\end{aligned}
$$

\subsection{The effective rate at which arriving customers are lost when finding the inventory level as zero}

Let $\eta_{I L}$ denote the effective rate at which arriving customers are lost when finding the inventory level as zero is given by

$$
\eta_{I L}=\sum_{i_{3}=0}^{N-1}(1-p)\left(N-i_{3}\right) \lambda \pi^{\left(0, S_{0}, i_{3}\right)}
$$

\section{Cost analysis and sensitivity investigation}

The expected total cost function per unit time is developed, wherein three decision variables $S, s$ and $N$ are considered. The objective is to find the optimum value of $S, s$ and $N$, simultaneously so that the cost function is minimized. First, the following cost elements are defined: The expected total cost per unit time (expected total cost rate) in the steady 
state for this model is defined so that

$c_{h}$ is the inventory carrying cost per unit item per unit time,

$c_{s}$ is the setup cost per order,

$c_{p}$ is the perishable cost per unit item per unit time,

$c_{w}$ is the waiting time cost of a customer in the orbit per unit time,

$c_{i}$ is the cost per interruption per unit time,

$c_{r}$ is the cost per repair per unit time,

$c_{l}$ is the shortage cost of a customer per unit time.

Using cost values $c_{h}, c_{s}, c_{p}, c_{w}, c_{i}, c_{r}$ and $c_{l}$ and the concept of crew-service equipment by White et al. [21], the total cost function $T C(S, s, N, J)$ per unit time in the steady state for this model is given by

$$
T C(S, s, N, J)=c_{h} \eta_{I}+c_{s} \eta_{R}+c_{p} \eta_{P}+c_{w} \eta_{C O}+c_{i} \eta_{I N T R}+c_{r} \eta_{P I}+c_{l} \eta_{C L}
$$

More clearly, $T C(S, s, N, J)=$

$$
\begin{aligned}
& c_{s} \sum_{i_{3}=0}^{N-1}\left\{\left(\sum_{k=0}^{J} r_{k} \mu_{0}\right)+s \gamma\right\} \phi^{\left(s+1, S_{1}, i_{3}\right)}+c_{s} \sum_{i_{3}=0}^{N}(s+1) \gamma \phi^{\left(s+1, S_{0}, i_{3}\right)}+c_{s} \sum_{i_{3}=0}^{N-1} s \gamma \phi^{\left(s+1, S_{2}, i_{3}\right)}+ \\
& c_{s} \sum_{i_{2}=1}^{2 J} \sum_{i_{3}=0}^{N-1}(s+1) \gamma \phi^{\left(s+1, i_{2}, i_{3}\right)}+c_{h} \sum_{i_{1}=1}^{S} i_{1} \phi^{\left(i_{1}\right)} \mathbf{e}+c_{p} \sum_{i_{1}=1}^{S} \sum_{i_{3}=0}^{N} i_{1} \gamma \phi^{\left(i_{1}, S_{0}, i_{3}\right)}+ \\
& c_{p} \sum_{i_{1}=1}^{S} \sum_{i=1}^{2} \sum_{i_{3}=0}^{N-1}\left(i_{1}-1\right) \gamma \phi^{\left(i_{1}, S_{i}, i_{3}\right)}+c_{p} \sum_{i_{1}=1}^{S} \sum_{i_{2}=1}^{2 J} \sum_{i_{3}=0}^{N-1} i_{1} \gamma \phi^{\left(i_{1}, i_{2}, i_{3}\right)}+c_{w} \sum_{i_{1}=0}^{S} \sum_{i_{3}=1}^{N} i_{3} \phi^{\left(i_{1}, S_{0}, i_{3}\right)}+ \\
& c_{w} \sum_{i_{1}=1}^{S} \sum_{i=1}^{2} \sum_{i_{3}=1}^{N-1} i_{3} \phi^{\left(i_{1}, S_{i}, i_{3}\right)}+c_{w} \sum_{i_{1}=0}^{S} \sum_{i_{2}=1}^{2 J} \sum_{i_{3}=1}^{N-1} i_{3} \phi^{\left(i_{1}, i_{2}, i_{3}\right)}+c_{i} \sum_{i_{1}=1}^{S} \sum_{i_{3}=0}^{N-1} \alpha_{0} \phi^{\left(i_{1}, S_{1}, i_{3}\right)}+ \\
& c_{i} \sum_{i_{1}=0}^{S} \sum_{i_{2}=1}^{N} \sum_{i_{3}=0}^{N-1} \alpha_{i_{2}} \phi^{\left(i_{1}, i_{2}, i_{3}\right)}+c_{r} \sum_{i_{1}=1}^{S} \sum_{i_{3}=0}^{N-1} \eta_{0} \phi^{\left(i_{1}, S_{2}, i_{3}\right)}+c_{r} \sum_{i_{1}=0}^{N-1} \sum_{i_{2}=J+1}^{N-1} \eta_{i_{3}=0}^{N} \eta_{\left(i_{2}-J\right)} \phi^{\left(i_{1}, i_{2}, i_{3}\right)}+ \\
& c_{l} \sum_{i_{3}=0}^{N-1}(1-p)\left(N-i_{3}\right) \lambda \phi^{\left(0, S_{0}, i_{3}\right)}+c_{l} \sum_{i_{1}=1}^{S} \sum_{i=1}^{2} \sum_{i_{3}=0}^{N-2}(1-p)\left(N-i_{3}\right) \lambda \phi^{\left(i_{1}, S_{i}, i_{3}\right)}+ \\
& c_{l} \sum_{i_{1}=0}^{S} \sum_{i_{2}=1}^{2 J} \sum_{i_{3}=0}^{N-2}(1-p)\left(N-i_{3}\right) \lambda \phi^{\left(i_{1}, i_{2}, i_{3}\right)} .
\end{aligned}
$$

A sensitivity investigation is given by considering the following parameters and cost values: $\lambda=0.04, \beta=5, \gamma=4, \theta=3, \mu_{0}=8, \mu_{1}=0.17, \mu_{2}=0.06, \mu_{3}=16, \alpha_{0}=0.05, \alpha_{1}=$ $0.08, \alpha_{2}=0.5, \alpha_{3}=0.7, \eta_{0}=4, \eta_{1}=11, \eta_{2}=6, \eta_{3}=0.06, r_{0}=0.25, r_{1}=$ $0.25, r_{2}=0.25, r_{3}=0.25, c_{h}=0.35, c_{s}=3, c_{p}=0.3, c_{w}=2, c_{l}=5, c_{i}=4, c_{r}=12$. The performance of the expected total cost function is investigated by allowing for any two variables to change, while fixing others at a constant level. Figure 2 reveals the 3dimensional plot of $T C(S, s)$. In Tables $1-3$, show the cost function of $T C(S, s, 10,3)$, 


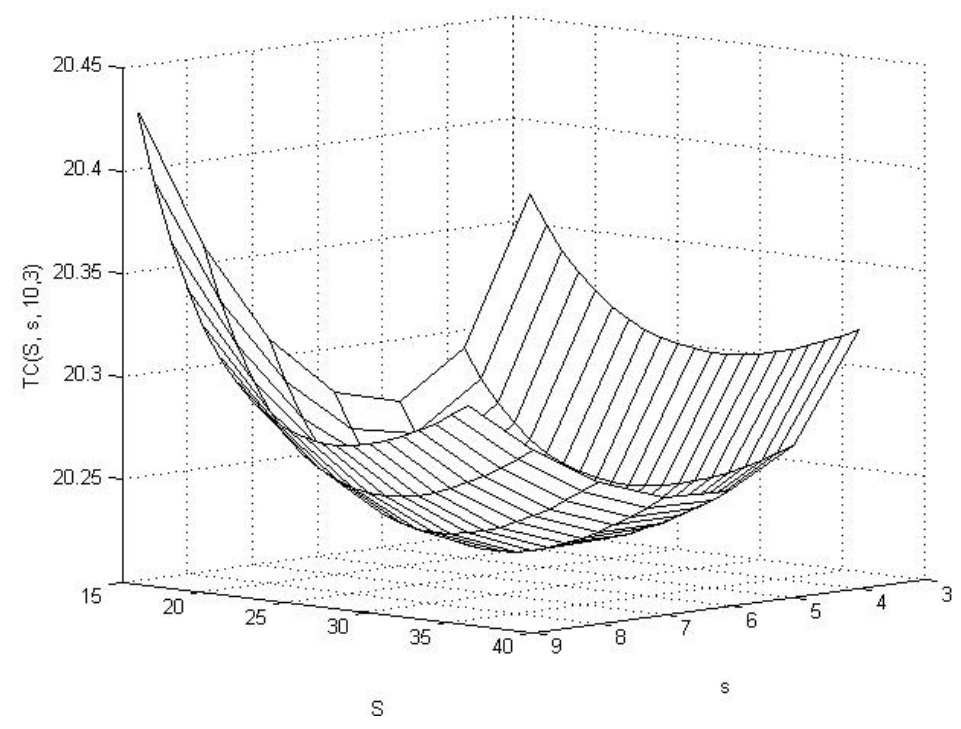

Figure 2: A three-dimensional plot of the cost function.

$T C(50, s, N, 3)$ and $T C(S, 6, N, 3)$ by fixed parameters and cost values as constant. After getting the Local Optima (LO) $S^{*}, s^{*}$ and $N^{*}$, the sensitivity investigation is carried out to observe how the changes in $S, s$ and $N$ affect the cost function. In Tables $1-3$, the values displayed in bold is the smallest value amongst the values in that row and likewise the values that are underlined is the smallest value amongst the values in that column. Therefore, a value (bold and underlined) shows a local minimum of $T C$. More clearly, varying $S$ and $s$, the optimal values are $S^{*}=30, s^{*}=6$ and $T C^{*}=20.213022$; varying $s$ and $N$, the optimal values are $s^{*}=8, N^{*}=14$ and $T C^{*}=9.264222$; varying $S$ and $N$, the optimal values are $S^{*}=34, N^{*}=12$ and $T C^{*}=7.821037$.

Next, a numerical illustration is performed to the optimal values $\left(S^{*}, s^{*}\right.$ and $\left.T C^{*}\right)$ based on changes in particular cost values. The numerical results are presented by considering the different cost values of $c_{h}, c_{s}, c_{p}, c_{w}, c_{l}, c_{i}$ and $c_{r}$. The following are observed from Tables $4-10$.

1. The optimal cost increases, when $c_{s}, c_{h}, c_{p}, c_{l}, c_{i}, c_{r}$ and $c_{w}$ increase. The optimal cost is more sensitive to $c_{w}$ than to $c_{s}, c_{h}, c_{p}, c_{i}, c_{r}$ and $c_{l}$.

2. As $c_{h}$ increases, as expected, the optimal values $s^{*}$ and $S^{*}$ decrease monotonically. This is expected since the holding cost increases, low stock is maintained in the inventory.

3. When $c_{l}$ and $c_{w}$ increase, the optimal values $s^{*}$ and $S^{*}$ increase monotonically. This is because if the waiting cost and shortage cost of a customer increase then high inventory is maintained to reduce the number of waiting (lost) customers.

4. If the setup cost $c_{s}$ increases, it is a common decision that more stock has to be maintained to avoid frequent ordering. This fact is also observed in the model.

5. It is noted that, if $c_{i}$ and $c_{r}$ increase, the optimal values of $s^{*}$ and $S^{*}$ increase monotonically and when the perishable $\operatorname{cost} c_{p}$ increases, $s^{*}$ and $S^{*}$ decrease monotonically. 


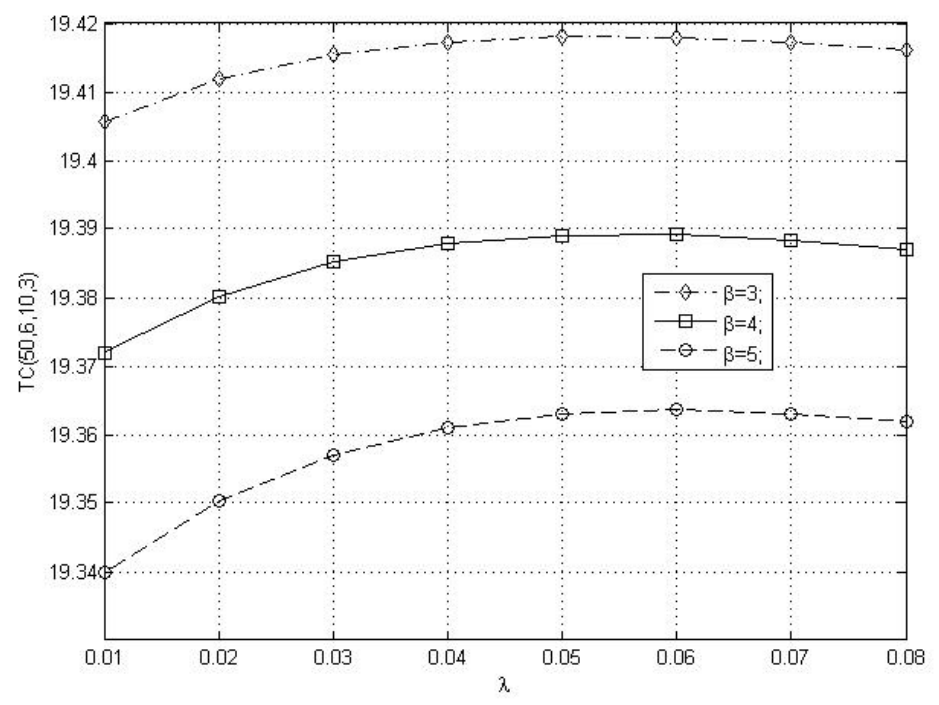

Figure 3: $\lambda$ vs $\beta$ on TC

Now, the impact of the parameter values $\lambda, \gamma, \beta, \theta, \mu_{0} \mu_{1}, \mu_{2}$ and $\mu_{3}$ on the total expected cost rate are looked at. From Figures $3-8$, the following is observed:

1. The optimal expected cost rate increases when $\lambda$ and $\gamma$ increase.

2. The optimal expected cost rate decreases when $\beta, \mu_{0}, \mu_{1}, \mu_{2}, \mu_{3}$ and $\theta$ increase.

The numerical illustrations are obtained by considering different service cases as follows:

Case 1: $r_{0}=0.25, r_{1}=0.25, r_{2}=0.25, r_{3}=0.25$;

Case 2: $r_{0}=0.4, r_{1}=0.2, r_{2}=0.2, r_{3}=0.2$;

Case 3: $r_{0}=0.2, r_{1}=0.4, r_{2}=0.2, r_{3}=0.2$;

Case $4: r_{0}=0.2, r_{1}=0.2, r_{2}=0.4, r_{3}=0.2$;

Case 5: $r_{0}=0.2, r_{1}=0.2, r_{2}=0.2, r_{3}=0.4$;

Case 6: $r_{0}=1, r_{1}=0, r_{2}=0, r_{3}=0$;

Case 7: $r_{0}=0.5, r_{1}=0.5, r_{2}=0, r_{3}=0$.

Now, the impact of parameter values $\lambda, \beta, \theta$ and different service cases (Cases 1-7) on $T C$ are looked at. From Figures $9-11$, the following is observed:

1. From Figures 9 and 10:

$T C_{\text {class } 4}>T C_{\text {class } 1}>T C_{\text {class } 3}>T C_{\text {class } 2}>T C_{\text {class } 5}>T C_{\text {class } 7}>T C_{\text {class } 6}$.

2. From Figure 11:

$T C_{\text {class } 4}>T C_{\text {class } 1}>T C_{\text {class } 2}>T C_{\text {class } 3}>T C_{\text {class } 5}>T C_{\text {class } 7}>T C_{\text {class } 6}$. 


\begin{tabular}{|l|l|l|l|l|l|}
\hline \multicolumn{1}{|c|}{$s$} & 4 & 5 & 6 & 7 & 8 \\
$S$ & & & & & \\
\hline 28 & 20.238179 & 20.214607 & $\mathbf{2 0 . 2 1 4 1 4 4}$ & 20.228737 & 20.254321 \\
\hline 29 & 20.237064 & $\underline{20.213788}$ & $\mathbf{2 0 . 2 1 3 0 9 0}$ & 20.226990 & 20.251429 \\
\hline 30 & $\underline{20.236783}$ & 20.213858 & $\underline{\mathbf{2 0 . 2 1 3 0 2 2}}$ & $\underline{20.226369}$ & 20.249854 \\
\hline 31 & 20.237253 & 20.214724 & $\mathbf{2 0 . 2 1 3 8 3 2}$ & 20.226743 & $\underline{20.249435}$ \\
\hline 32 & 20.238399 & 20.216305 & $\mathbf{2 0 . 2 1 5 4 2 6}$ & 20.228002 & 20.250036 \\
\hline
\end{tabular}

Table 1: Total expected cost rate as a function of $S$ and $s$

\begin{tabular}{|c|c|c|c|c|c|}
\hline$N^{s}$ & 6 & 7 & 8 & 9 & 10 \\
\hline 12 & 9.475022 & 9.375660 & 9.264644 & 9.344231 & 9.356036 \\
\hline 13 & 9.474643 & 9.375323 & 9.264346 & 9.343971 & 9.355811 \\
\hline 14 & 9.474447 & 9.375164 & 9.264222 & 9.343879 & 9.355750 \\
\hline 15 & 9.474402 & 9.375149 & 9.264237 & 9.343923 & 9.355822 \\
\hline 16 & 9.474479 & 9.375255 & 9.264369 & 9.344080 & 9.356003 \\
\hline
\end{tabular}

Table 2: Total expected cost rate as a function of $s$ and $N$

\begin{tabular}{|c|c|c|c|c|c|}
\hline$N$ & 10 & 11 & 12 & 13 & 14 \\
\hline 32 & 7.900699 & 7.847420 & 7.827450 & 7.834535 & 7.869472 \\
\hline 33 & 7.882873 & 7.836107 & 7.822051 & 7.834425 & 7.868038 \\
\hline 34 & 7.870182 & 7.829554 & 7.821037 & $\overline{7.838337}$ & 7.866280 \\
\hline 35 & 7.862038 & 7.8272 & $\overline{7.82}$ & 7.845770 & $\overline{7.877730}$ \\
\hline 63 & 7.877938 & 7.828566 & 7.830121 & 7.856304 & 7.891997 \\
\hline
\end{tabular}

Table 3: Total expected cost rate as a function of $S$ and $N$ 


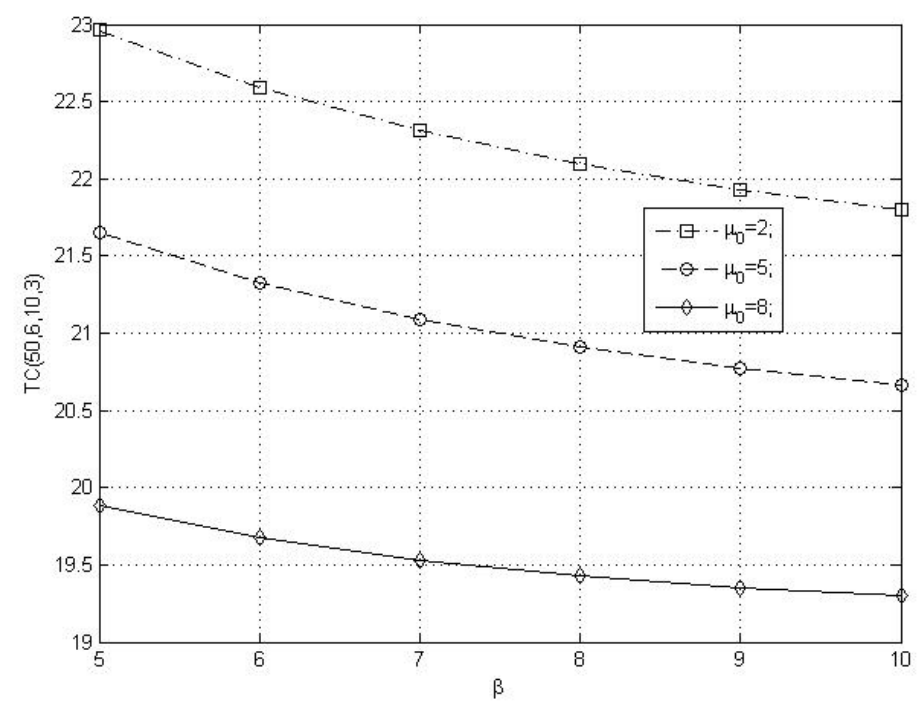

Figure 4: $\beta$ vs $\mu_{0}$ on TC

\begin{tabular}{|c|c|c|c|c|c|c|c|c|c|c|}
\hline$c^{c_{h}} c_{s}$ & \multicolumn{2}{|c|}{1.5} & \multicolumn{2}{|r|}{2} & \multicolumn{2}{|c|}{2.5} & \multicolumn{2}{|c|}{3} & \multicolumn{2}{|c|}{3.5} \\
\hline \multirow[t]{2}{*}{0.25} & 50 & 6 & 56 & 6 & 59 & 5 & 62 & 4 & 65 & 4 \\
\hline & \multicolumn{2}{|c|}{21.365796} & \multicolumn{2}{|c|}{21.534480} & \multicolumn{2}{|c|}{21.690773} & \multicolumn{2}{|c|}{21.837029} & \multicolumn{2}{|c|}{21.968881} \\
\hline \multirow[t]{2}{*}{0.30} & 47 & 6 & 51 & 6 & 56 & 5 & 60 & 4 & 63 & 4 \\
\hline & \multicolumn{2}{|c|}{21.378759} & \multicolumn{2}{|c|}{21.546029} & \multicolumn{2}{|c|}{21.701847} & \multicolumn{2}{|c|}{21.847088} & \multicolumn{2}{|c|}{21.978081} \\
\hline \multirow[t]{2}{*}{0.35} & 45 & 5 & 48 & 5 & 51 & 5 & 56 & 4 & 60 & 4 \\
\hline & \multicolumn{2}{|c|}{21.391656} & \multicolumn{2}{|c|}{21.557579} & \multicolumn{2}{|c|}{21.712886} & \multicolumn{2}{|c|}{21.857099} & \multicolumn{2}{|c|}{21.988377} \\
\hline \multirow[t]{2}{*}{0.40} & 40 & 5 & 44 & 5 & 48 & 4 & 51 & 3 & 56 & 3 \\
\hline & \multicolumn{2}{|c|}{21.404441} & \multicolumn{2}{|c|}{21.569125} & \multicolumn{2}{|c|}{21.723925} & \multicolumn{2}{|c|}{21.867110} & \multicolumn{2}{|c|}{21.998115} \\
\hline \multirow[t]{2}{*}{0.45} & 36 & 4 & 40 & 4 & 44 & 4 & 48 & 3 & 52 & 3 \\
\hline & \multicolumn{2}{|c|}{21.417227} & \multicolumn{2}{|c|}{21.580540} & \multicolumn{2}{|c|}{21.734964} & \multicolumn{2}{|c|}{21.877121} & \multicolumn{2}{|c|}{22.007852} \\
\hline
\end{tabular}

Table 4: Variation in optimal values for different values of $c_{h}$ and $c_{s}$ with $c_{p}=0.3, c_{w}=$ $2, c_{l}=5, c_{i}=4, c_{r}=12$.

\begin{tabular}{|c|c|c|c|c|c|c|c|c|c|c|}
\hline$c_{h} c_{p}$ & \multicolumn{2}{|c|}{0.1} & \multicolumn{2}{|c|}{0.2} & \multicolumn{2}{|c|}{0.3} & \multicolumn{2}{|c|}{0.4} & \multicolumn{2}{|c|}{0.5} \\
\hline \multirow[t]{2}{*}{0.25} & 50 & 6 & 48 & 5 & 45 & 5 & 40 & 4 & 35 & 3 \\
\hline & \multicolumn{2}{|c|}{21.365796} & \multicolumn{2}{|c|}{22.257257} & \multicolumn{2}{|c|}{22.919692} & \multicolumn{2}{|c|}{23.487756} & \multicolumn{2}{|c|}{23.988367} \\
\hline \multirow[t]{2}{*}{0.30} & 47 & 6 & 46 & 5 & 40 & 5 & 36 & 4 & 33 & 3 \\
\hline & \multicolumn{2}{|c|}{21.378759} & \multicolumn{2}{|c|}{22.273638} & \multicolumn{2}{|c|}{22.937827} & \multicolumn{2}{|c|}{23.508647} & \multicolumn{2}{|c|}{24.007861} \\
\hline \multirow[t]{2}{*}{0.35} & 45 & 5 & 40 & 4 & 35 & 4 & 32 & 4 & 30 & 3 \\
\hline & \multicolumn{2}{|c|}{21.391656} & \multicolumn{2}{|c|}{22.289913} & \multicolumn{2}{|c|}{$\frac{1}{22.955962}$} & \multicolumn{2}{|c|}{22.528934} & \multicolumn{2}{|c|}{24.027356} \\
\hline \multirow[t]{2}{*}{0.40} & 40 & 5 & 36 & 4 & 30 & 4 & 27 & 3 & 24 & 3 \\
\hline & \multicolumn{2}{|c|}{21.404441} & \multicolumn{2}{|c|}{22.306188} & \multicolumn{2}{|c|}{22.973757} & \multicolumn{2}{|c|}{23.549029} & \multicolumn{2}{|c|}{24.046851} \\
\hline \multirow[t]{2}{*}{0.45} & 36 & 4 & 30 & 4 & 24 & 4 & 20 & 3 & 18 & 3 \\
\hline & \multicolumn{2}{|c|}{21.417227} & \multicolumn{2}{|c|}{22.321962} & \multicolumn{2}{|c|}{22.991351} & \multicolumn{2}{|c|}{$\begin{array}{l}23.569123 \\
\end{array}$} & \multicolumn{2}{|c|}{24.066346} \\
\hline
\end{tabular}

Table 5: Variation in optimal values for different values of $c_{h}$ and $c_{p}$ with $c_{s}=3, c_{w}=$ $2, c_{l}=5, c_{i}=4, c_{r}=12$. 


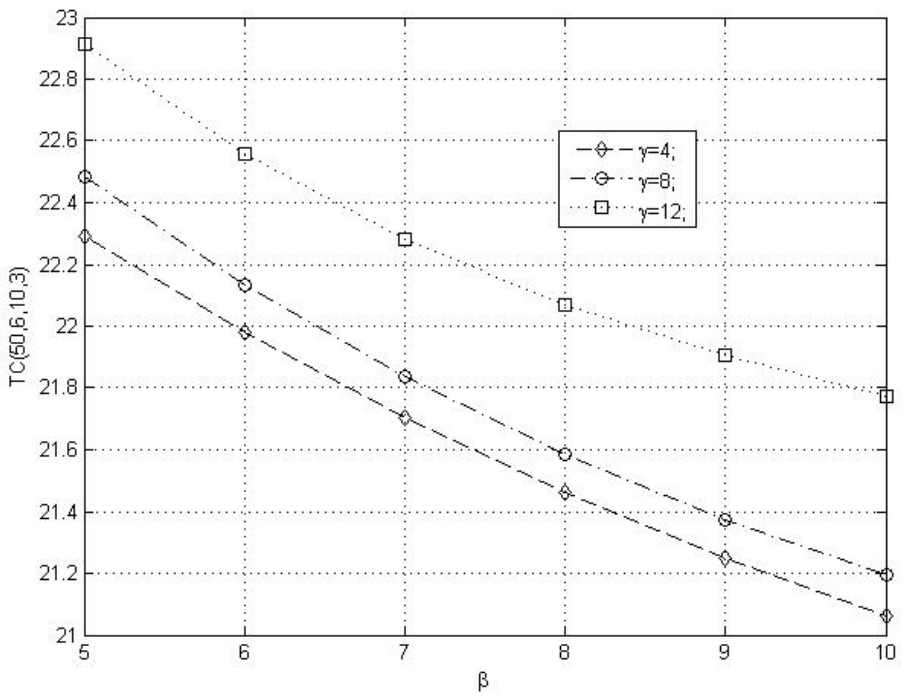

Figure 5: $\beta$ vs $\gamma$ on TC.

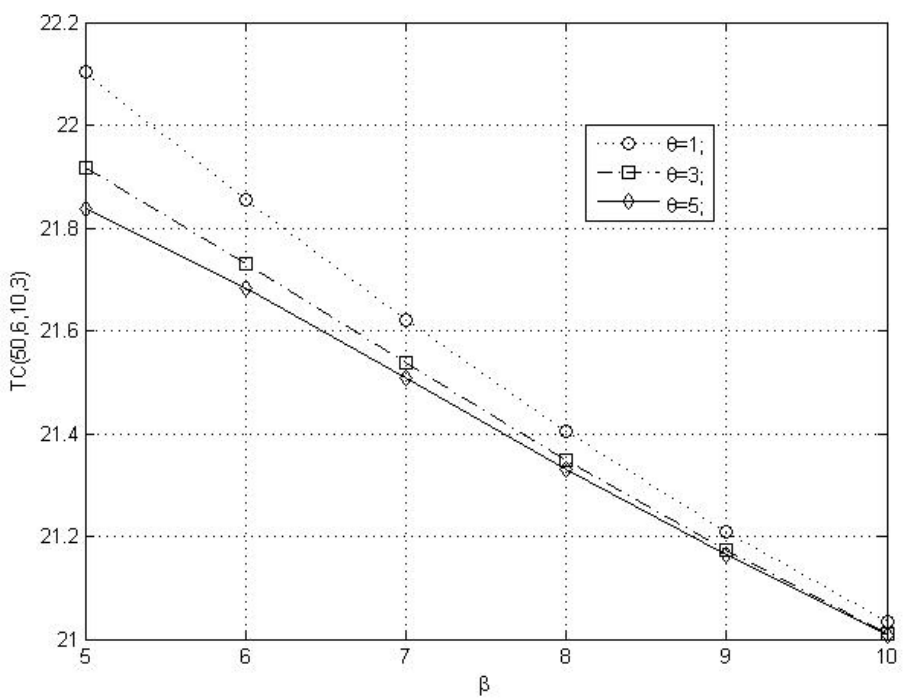

Figure $6: \beta$ vs $\theta$ on TC. 


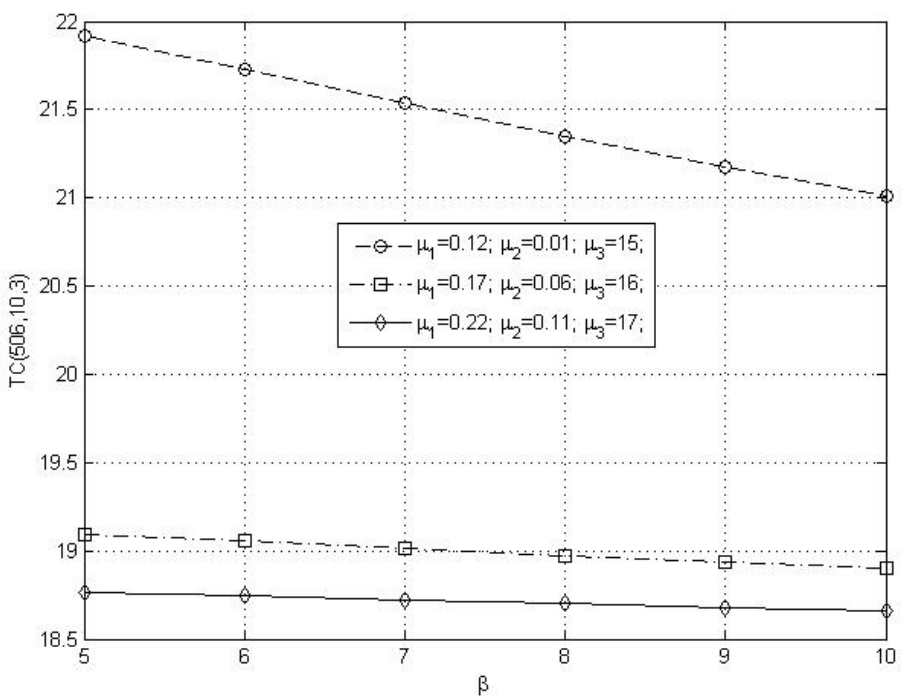

Figure $7: \beta$ vs $\mu_{1}, \mu_{2}, \mu_{3}$ on TC.

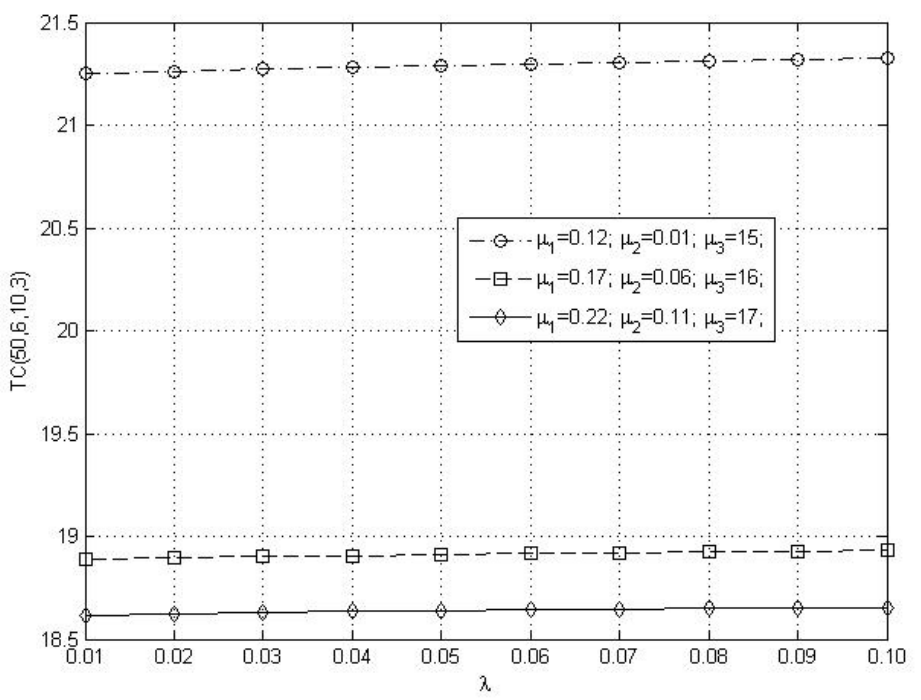

Figure 8: $\beta$ vs $\mu_{1}, \mu_{2}, \mu_{3}$ on TC. 


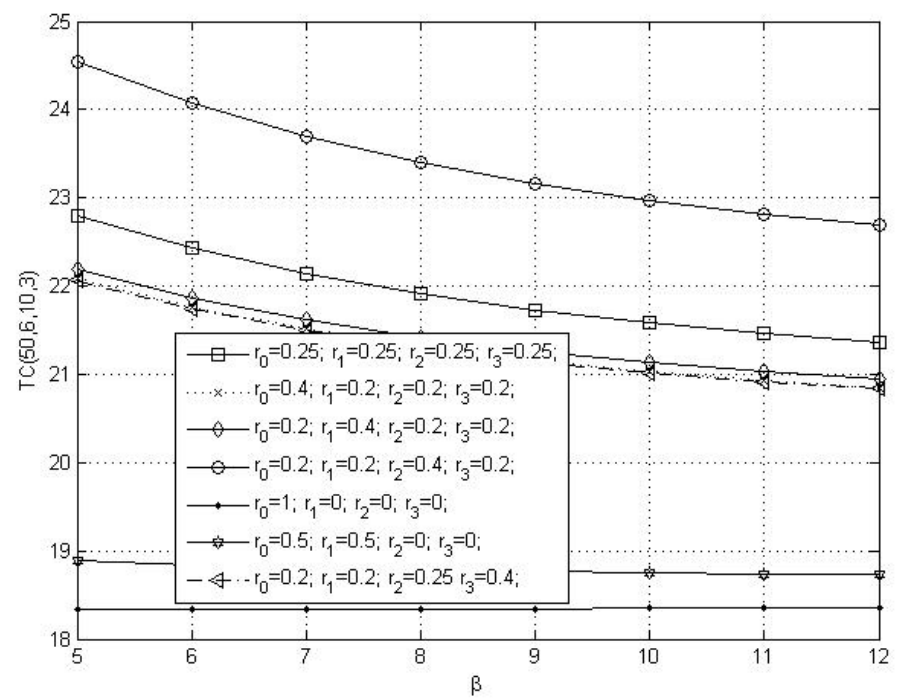

Figure 9: $\beta$ vs service cases on TC.

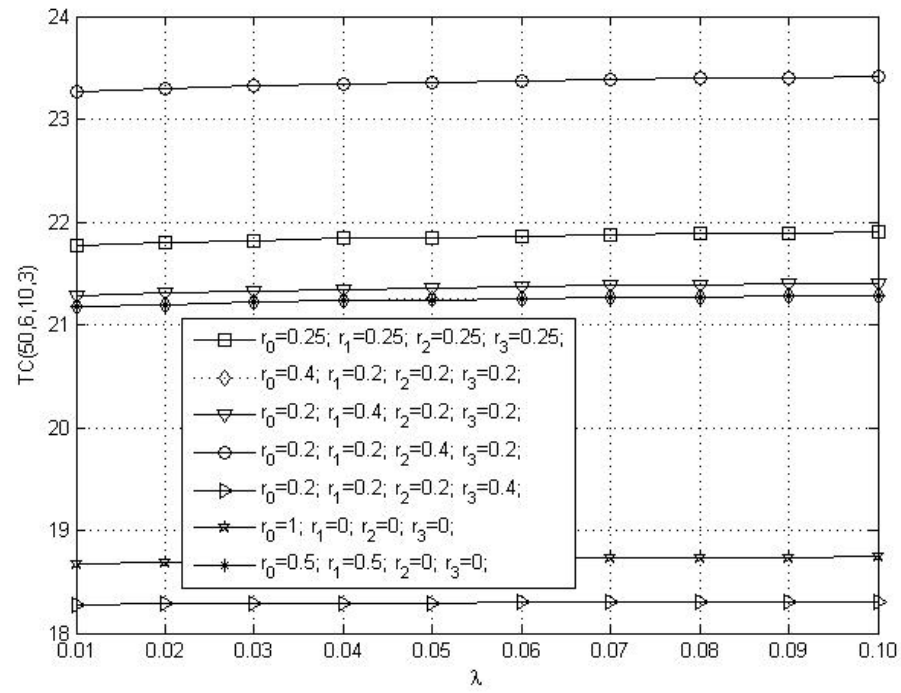

Figure 10: $\beta$ vs service cases on TC. 


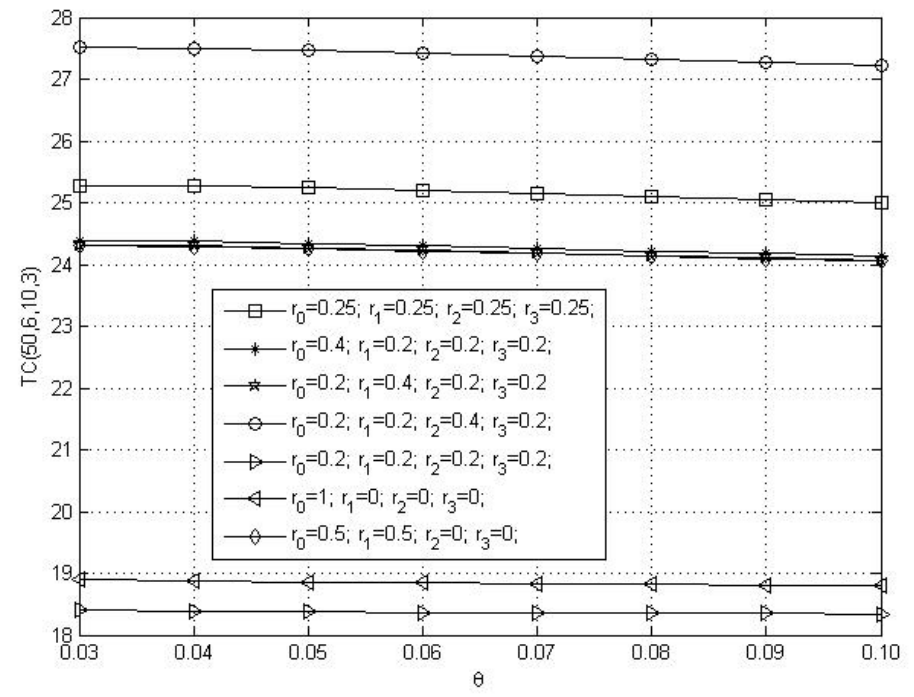

Figure 11: $\beta$ vs service cases on TC.

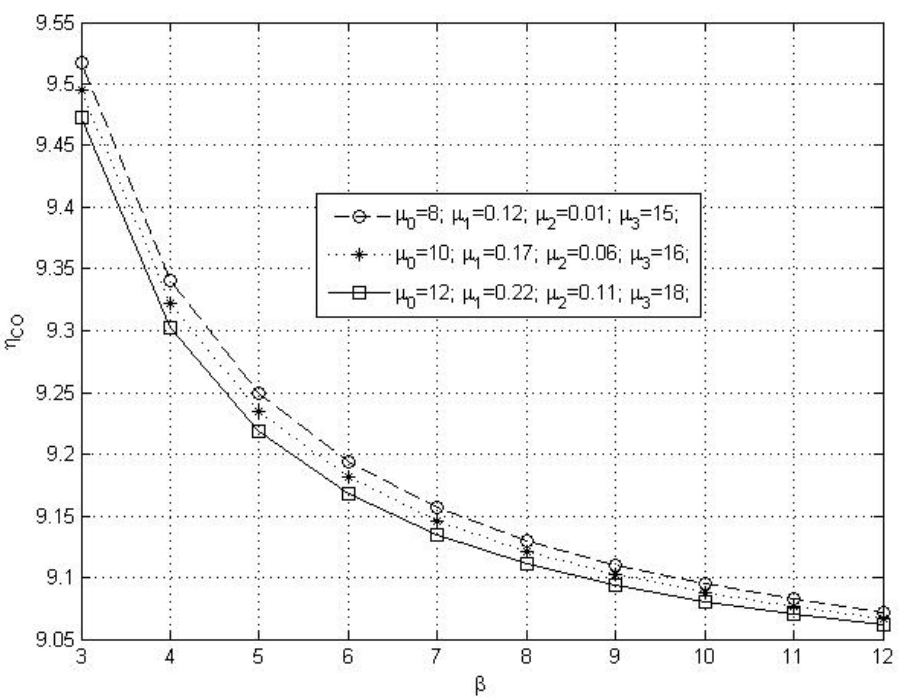

Figure 12 : $\beta$ vs $\mu_{0}, \mu_{1}, \mu_{2}$ and $\mu_{3}$ on $\eta_{C O}$. 


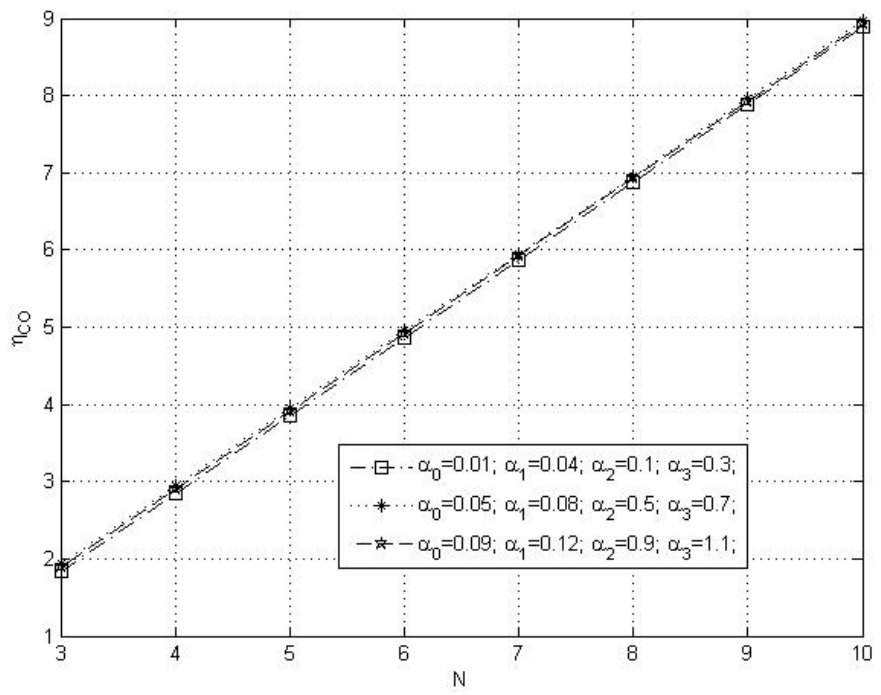

Figure 13: $N$ vs $\alpha_{0}, \alpha_{1}, \alpha_{2}, \alpha_{3}$ on $\eta_{C O}$.

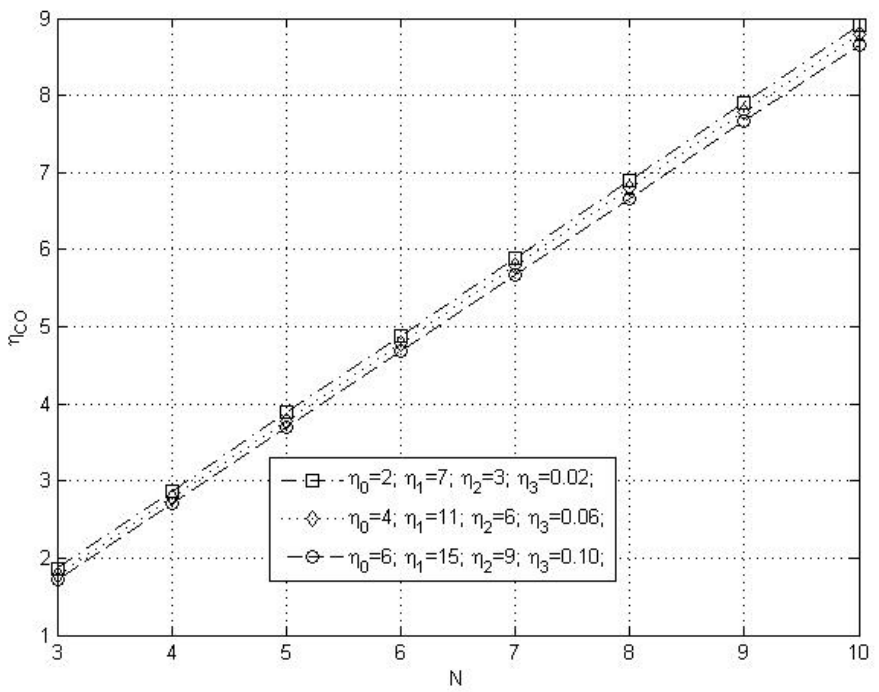

Figure 14: $N$ vs $\eta_{0}, \eta_{1}, \eta_{2}, \eta_{3}$ on $\eta_{C O}$. 


\begin{tabular}{|c|c|c|c|c|c|c|c|c|c|c|}
\hline$c_{h}^{c_{w}}$ & \multicolumn{2}{|c|}{2} & \multicolumn{2}{|c|}{3} & \multicolumn{2}{|r|}{4} & \multicolumn{2}{|c|}{5} & \multicolumn{2}{|r|}{6} \\
\hline \multirow[t]{2}{*}{0.25} & 50 & 6 & 54 & 6 & 55 & 7 & 56 & 8 & 57 & 8 \\
\hline & \multicolumn{2}{|c|}{21.365796} & \multicolumn{2}{|c|}{30.361160} & \multicolumn{2}{|c|}{39.356524} & \multicolumn{2}{|c|}{48.351888} & \multicolumn{2}{|c|}{57.347252} \\
\hline \multirow[t]{2}{*}{0.30} & 47 & 6 & 50 & 6 & 52 & \begin{tabular}{|l|}
7 \\
\end{tabular} & 53 & 7 & 54 & 7 \\
\hline & \multicolumn{2}{|c|}{21.378759} & \multicolumn{2}{|c|}{30.374123} & \multicolumn{2}{|c|}{39.369487} & \multicolumn{2}{|c|}{48.364851} & \multicolumn{2}{|c|}{57.360215} \\
\hline \multirow[t]{2}{*}{0.35} & 45 & 5 & 48 & 5 & 50 & \begin{tabular}{|l|}
6 \\
\end{tabular} & 51 & 6 & 53 & 7 \\
\hline & \multicolumn{2}{|c|}{21.391656} & \multicolumn{2}{|c|}{30.387039} & \multicolumn{2}{|c|}{39.382423} & \multicolumn{2}{|c|}{48.377807} & \multicolumn{2}{|c|}{57.373178} \\
\hline \multirow{2}{*}{0.40} & 40 & 5 & 45 & 5 & 48 & 5 & 49 & 6 & 50 & 6 \\
\hline & \multicolumn{2}{|c|}{21.404441} & \multicolumn{2}{|c|}{30.399825} & \multicolumn{2}{|c|}{39.395209} & \multicolumn{2}{|c|}{48.390593} & \multicolumn{2}{|c|}{57.385976} \\
\hline \multirow[t]{2}{*}{0.45} & 36 & 4 & 40 & 4 & 45 & 5 & & 5 & & 6 \\
\hline & \multicolumn{2}{|c|}{21.417227} & \multicolumn{2}{|c|}{30.412611} & \multicolumn{2}{|c|}{39.407995} & \multicolumn{2}{|c|}{48.403379} & \multicolumn{2}{|c|}{57.398762} \\
\hline
\end{tabular}

Table 6: Variation in optimal values for different values of $c_{h}$ and $c_{w}$ with $c_{s}=3, c_{p}=$ $0.3, c_{l}=5, c_{i}=4, c_{r}=12$.

\begin{tabular}{|c|c|c|c|c|c|c|c|c|c|c|}
\hline$c_{s}{ }^{c_{p}}$ & \multicolumn{2}{|c|}{0.1} & \multicolumn{2}{|c|}{0.2} & \multicolumn{2}{|c|}{0.3} & \multicolumn{2}{|c|}{0.4} & \multicolumn{2}{|c|}{0.5} \\
\hline \multirow[t]{2}{*}{1.5} & 50 & 6 & 48 & 5 & 45 & 5 & 40 & 4 & 35 & 3 \\
\hline & \multicolumn{2}{|c|}{21.365796} & \multicolumn{2}{|c|}{22.257257} & \multicolumn{2}{|c|}{22.919692} & \multicolumn{2}{|c|}{23.487756} & \multicolumn{2}{|c|}{23.988367} \\
\hline \multirow[t]{2}{*}{2} & 54 & 4 & 52 & 4 & 48 & 4 & 45 & 3 & 40 & 3 \\
\hline & \multicolumn{2}{|c|}{21.534480} & \multicolumn{2}{|c|}{22.457181} & \multicolumn{2}{|c|}{23.164813} & \multicolumn{2}{|c|}{23.738541} & \multicolumn{2}{|c|}{24.235497} \\
\hline \multirow[t]{2}{*}{2.5} & 57 & 4 & 55 & 4 & 52 & 3 & 50 & 3 & 47 & 2 \\
\hline & \multicolumn{2}{|c|}{21.690773} & \multicolumn{2}{|c|}{22.642965} & \multicolumn{2}{|c|}{23.371365} & \multicolumn{2}{|c|}{23.953040} & \multicolumn{2}{|c|}{24.471896} \\
\hline \multirow[t]{2}{*}{3} & 60 & 3 & 57 & 3 & 55 & 3 & 52 & 2 & 50 & 2 \\
\hline & \multicolumn{2}{|c|}{21.837029} & \multicolumn{2}{|c|}{22.821527} & \multicolumn{2}{|c|}{23.554849} & \multicolumn{2}{|c|}{24.159996} & \multicolumn{2}{|c|}{24.699138} \\
\hline \multirow[t]{2}{*}{3.5} & 63 & 3 & 60 & 3 & 59 & 3 & 57 & 2 & 53 & 2 \\
\hline & \multicolumn{2}{|c|}{21.968881} & \multicolumn{2}{|c|}{22.993137} & \multicolumn{2}{|c|}{23.733211} & \multicolumn{2}{|c|}{24.360297} & \multicolumn{2}{|c|}{24.918487} \\
\hline
\end{tabular}

Table 7: Variation in optimal values for different values of $c_{s}$ and $c_{p}$ with $c_{h}=0.35, c_{w}=$ $2, c_{l}=5, c_{i}=4, c_{r}=12$.

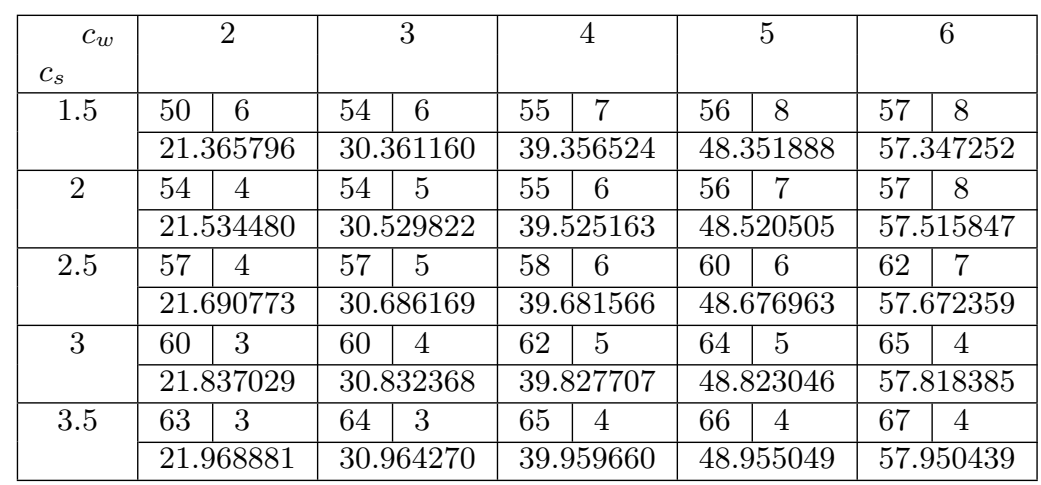

Table 8: Variation in optimal values for different values of $c_{s}$ and $c_{w}$ with $c_{h}=0.35, c_{p}=$ $0.3, c_{l}=5, c_{i}=4, c_{r}=12$. 


\begin{tabular}{|c|c|c|c|c|c|c|c|c|c|c|}
\hline$c_{s}{ }^{c_{l}}$ & \multicolumn{2}{|c|}{5} & \multicolumn{2}{|c|}{6} & \multicolumn{2}{|c|}{7} & \multicolumn{2}{|c|}{8} & \multicolumn{2}{|c|}{9} \\
\hline \multirow[t]{2}{*}{1.5} & 42 & 5 & 47 & 6 & 50 & 6 & 52 & 7 & 55 & 8 \\
\hline & \multicolumn{2}{|c|}{21.273175} & \multicolumn{2}{|c|}{21.319551} & \multicolumn{2}{|c|}{ 21.365796 } & \multicolumn{2}{|c|}{21.412040} & \multicolumn{2}{|c|}{21.458179} \\
\hline \multirow[t]{2}{*}{2} & 45 & 4 & 50 & 4 & 54 & 4 & 58 & 6 & 60 & 7 \\
\hline & \multicolumn{2}{|c|}{21.441252} & \multicolumn{2}{|c|}{21.487907} & \multicolumn{2}{|c|}{21.534480} & \multicolumn{2}{|c|}{21.580947} & \multicolumn{2}{|c|}{21.627414} \\
\hline \multirow[t]{2}{*}{2.5} & 49 & 4 & 53 & 4 & 57 & 4 & 60 & 5 & 64 & 6 \\
\hline & \multicolumn{2}{|c|}{21.598933} & \multicolumn{2}{|c|}{21.644853} & \multicolumn{2}{|c|}{21.690773} & \multicolumn{2}{|c|}{21.736552} & \multicolumn{2}{|c|}{21.782295} \\
\hline \multirow[t]{2}{*}{3} & 54 & 3 & 59 & 3 & 60 & 3 & 64 & 4 & 69 & 5 \\
\hline & \multicolumn{2}{|c|}{21.744041} & \multicolumn{2}{|c|}{21.790535} & \multicolumn{2}{|c|}{21.837029} & \multicolumn{2}{|c|}{21.883401} & \multicolumn{2}{|c|}{21.929725} \\
\hline \multirow[t]{2}{*}{3.5} & 58 & 3 & 61 & 3 & 63 & 3 & & 3 & 72 & 4 \\
\hline & \multicolumn{2}{|c|}{21.876897} & \multicolumn{2}{|c|}{21.922889} & \multicolumn{2}{|c|}{21.968881} & \multicolumn{2}{|c|}{22.014732} & \multicolumn{2}{|c|}{22.060561} \\
\hline
\end{tabular}

Table 9: Variation in optimal values for different values of $c_{s}$ and $c_{l}$ with $c_{h}=0.35, c_{p}=$ $0.3, c_{w}=2, c_{i}=4, c_{r}=12$.

\begin{tabular}{|c|c|c|c|c|c|c|c|c|c|c|}
\hline $\begin{array}{ll} & c_{r} \\
c_{i}\end{array}$ & \multicolumn{2}{|c|}{3} & \multicolumn{2}{|c|}{4} & \multicolumn{2}{|c|}{5} & \multicolumn{2}{|c|}{6} & \multicolumn{2}{|c|}{7} \\
\hline & 42 & 5 & 44 & 6 & 45 & 6 & 46 & 6 & 47 & 7 \\
\hline & \multicolumn{2}{|c|}{21.205227} & \multicolumn{2}{|c|}{21.365796} & \multicolumn{2}{|c|}{$\frac{1}{21.522320}$} & \multicolumn{2}{|c|}{ 21.678341 } & \multicolumn{2}{|c|}{21.833571} \\
\hline \multirow[t]{2}{*}{12} & 45 & 6 & 46 & 6 & 47 & 6 & 48 & 7 & 49 & 7 \\
\hline & \multicolumn{2}{|c|}{21.347984} & \multicolumn{2}{|c|}{21.504086} & \multicolumn{2}{|c|}{21.659696} & \multicolumn{2}{|c|}{21.810874} & \multicolumn{2}{|c|}{21.959569} \\
\hline \multirow[t]{2}{*}{14} & 53 & 6 & 54 & 7 & 55 & 7 & 56 & 7 & 57 & 7 \\
\hline & \multicolumn{2}{|c|}{21.485517} & \multicolumn{2}{|c|}{21.639196} & \multicolumn{2}{|c|}{ 21.787930 } & \multicolumn{2}{|c|}{ 21.936342 } & \multicolumn{2}{|c|}{22.084391} \\
\hline \multirow[t]{2}{*}{16} & 55 & 7 & 56 & 7 & 56 & 7 & 57 & 7 & 58 & 8 \\
\hline & \multicolumn{2}{|c|}{21.616291} & \multicolumn{2}{|c|}{21.764736} & \multicolumn{2}{|c|}{21.912784} & \multicolumn{2}{|c|}{22.060517} & \multicolumn{2}{|c|}{22.203573} \\
\hline \multirow[t]{2}{*}{18} & 56 & 7 & 57 & 7 & 58 & 8 & 59 & 8 & 60 & 8 \\
\hline & \multicolumn{2}{|c|}{21.741181} & \multicolumn{2}{|c|}{21.888943} & \multicolumn{2}{|c|}{$\begin{array}{l}22.033663 \\
\end{array}$} & \multicolumn{2}{|c|}{22.176304} & \multicolumn{2}{|c|}{22.318753} \\
\hline
\end{tabular}

Table 10: Variation in optimal values for different values of $c_{r}$ and $c_{i}$ with $c_{h}=0.35, c_{s}=$ $3, c_{p}=0.3, c_{w}=2, c_{l}=5$.

Finally, the impact of the parameters $\lambda, \beta, \mu_{0}, \mu_{1}, \mu_{2}, \mu_{3}, \eta_{0}, \eta_{1}, \eta_{2}, \eta_{3}, \alpha_{0}, \alpha_{1}, \alpha_{2}, \alpha_{3}$ and $N$ on the expected number of customers in orbit $\eta_{C O}$ are studied. The following is observed from Figures 12-14.

1. If $\beta, \mu_{i}$ and $\eta_{i}, i=0,1,2,3$ increase, then the expected number of customers in the orbit $\eta_{C O}$ decreases.

2. When $N$ and $\alpha_{i}, i=0,1,2,3$, increase, then $\eta_{C O}$ increases.

\section{Conclusion}

In this paper, a continuous review stochastic inventory system with $J$ additional options for service, server interruptions, returning customers and finite populations were analyzed. The stationary distribution of the number of customers in the orbit and the inventory level is obtained by matrix method. Various system performance measures are derived and the long-run total expected cost rate is calculated and also the Laplace-Stieltjes transform of the waiting time of the orbiting customer is derived. By assuming a suitable cost structure on the inventory system, extensive numerical illustrations were presented to show the effect of change of values for constants on the total expected cost rate. 


\section{Acknowledgements}

The second author's research was supported by The University of Madras through C.5/DSTPURSE Phase II/2014/686. The authors would like to express their thankfulness to the the chief editor and anonymous referees for precious remarks, which very much improved the excellence of this paper.

\section{References}

[1] Artalejo JR, 1998, Retrial queues with a finite number of sources, Journal of the Korean Mathematical Society, 35(3), pp. 503-525.

[2] Artalejo JR, Krishnamoorthy A \& Lopez-Herrero MJ, 2006, Numerical analysis of (s, $s$ ) inventory systems with repeated attempts, Annals of Operations Research, 141(1), pp. 67-83.

[3] Berman O, Kaplan EH \& Shevishak DG, 1993, Deterministic approximations for inventory management at service facilities, IIE Transactions, 25(5), pp. 98-104.

[4] Berman O \& Kim E, 1999, Stochastic models for inventory management at service facilities, Stochastic Models, 15(4), pp. 695-718.

[5] Berman O \& Kim E, 2004, Dynamic inventory strategies for profit maximization in a service facility with stochastic service, demand and lead time, Mathematical methods of operations research, 60(3), pp. $497-521$.

[6] Berman O \& Sapna K, 2000, Inventory management at service facilities for systems with arbitrarily distributed service times, Stochastic Models, 16(3), pp. 343-360.

[7] Falin G \& Artalejo JR, 1998, A finite source retrial queue, European Journal of Operational Research, 108(2), pp. 409-424.

[8] Falin G \& Templeton JG, 1997, Retrial queues, volume 75, CRC Press, Chapman \& Hall, New York (NY).

[9] Jeganathan K, 2015, A single server perishable inventory system with $n$ additional options for service, Journal of Mathematical Modeling, 2(2), pp. 187-216.

[10] Jeganathan K, Kathiresan J \& Anbazhagan N, 2016, A retrial inventory system with priority customers and second optional service, OPSEARCH, 53(4), pp. 808-834.

[11] Ke JC, Wu CH \& PEARN WL, 2011, Multi-server retrial queue with second optional service: algorithmic computation and optimisation, International Journal of Systems Science, 42(10), pp. 1755-1769.

[12] Krishnamoorthy A, Nair SS \& Narayanan VC, 2010, An inventory model with server interruptions, Proceedings of the 5 th International Conference on Queueing Theory and Network Applications, 24-26 July 2010, Beijing, China, pp. 132-139.

[13] Krishnamoorthy A, Nair SS \& Narayanan VC, 2012, An inventory model with server interruptions and retrials, Operational Research, 12(2), pp. 151-171.

[14] Kulkarni VG, 2016, Modeling and analysis of stochastic systems, CRC Press, Chapman \& Hall, New York (NY).

[15] Lawrence AS, Sivakumar B \& Arivarignan G, 2013, A perishable inventory system with service facility and finite source, Applied Mathematical Modelling, 37(7), pp. 4771-4786.

[16] Schwarz M, Sauer C, Daduna H, Kulik R \& Szekli R, 2006, M/m/1 queueing systems with inventory, Queueing Systems, 54(1), pp. 55-78. 
[17] Sigman K \& Simchi-Levi D, 1992, Light traffic heuristic for anm/g/1 queue with limited inventory, Annals of Operations Research, 40(1), pp. 371-380.

[18] Sivakumar B, 2009, A perishable inventory system with retrial demands and a finite population, Journal of Computational and Applied Mathematics, 224(1), pp. 29-38.

[19] Wang J \& Li J, 2009, A single server retrial queue with general retrial times and two-phase service, Journal of Systems Science and Complexity, 22(2), pp. 291-302.

[20] Wang J \& Zhao Q, 2007, A discrete-time geo/g/1 retrial queue with starting failures and second optional service, Computers \& Mathematics with Applications, 53(1), pp. 115-127.

[21] White JA, 2012, Analysis of queueing systems, Academic Press, New York (NY).

[22] Yadavalli V, Anbazhagan N \& Jeganathan K, 2015, A two heterogeneous servers perishable inventory system of a finite population with one unreliable server and repeated attempts, Pakistan Journal of Statistics, 31(1), pp. 135-158.

[23] Yadavalli V \& Jeganathan K, 2016, A finite source perishable inventory system with second optional service and server interruptions, ORiON, 32(1), pp. 23-53.

[24] Yadavalli V, Sivakumar B, Arivarignan G \& Adetunji O, 2012, A finite source multi-server inventory system with service facility, Computers \& Industrial Engineering, 63(4), pp. 739-753. 